\title{
Chapter 4: Direct Numerical Simulations for Combustion Science: Past, Present, and Future
}

\author{
Hong G. Im \\ ${ }^{1}$ Clean Combustion Research Center, King Abdullah University of Science and Technology, \\ Thuwal 23955, Saudi Arabia
}

\section{Introduction}

It has been nearly three decades since direct numerical simulations (DNS) were used in combustion research. First, a disclaimer on terminology is warranted. The word "direct" herein refers to the absence of closure submodels in the context of traditional turbulent flow simulations, such as the statistical average of the Reynolds stress terms (in case of Reynoldsaveraged Navier-Stokes, RANS) or the subgrid stress terms (in case of large eddy simulation, LES). The same cannot be said for the chemical reaction source terms appearing in the conservation equations for reacting flows, as essentially all reaction source terms represented in the current DNS are a continuum representation of the chemical reaction events that result from inter-molecular collisions. As such, the "DNS" designation is only valid in multidimensional, turbulent flow simulations in which all relevant continuum scales, including the Kolmogorov scale eddies and flame structures, are fully resolved.

The role of DNS in combustion research continues to expand with the advances in computing power, which allows large DNS simulations approaching the parametric conditions relevant to realistic laboratory conditions. Given that the thermo-chemical and transport properties of individual reactive scalars are well known, and that the computational solution algorithm is robust and all scales are sufficiently resolved, the solution fields generated by DNS are spatially and temporally accurate. Contributions of DNS in fundamental combustion science are twofold. First, the solutions provide detailed information of flow-chemistry interaction for highly localized phenomena, such as flame structure changes, quenching, reignition, and edge flame formation. Secondly, the simulations that are run in a sufficiently large domain size and a long physical time serve as a useful benchmark for validating turbulent combustion submodels by allowing statistical information of key observables. In this chapter, some major accomplishments of DNS in these two aspects will be reviewed in chronological order. Emphasis will be on the first contributions, the scientific discoveries, while detailed description of the latter will be left for other dedicated chapters.

\section{A Brief History of Early Combustion DNS}

\subsection{From Nonreacting to Reacting Flow DNS}

Historical aspects of DNS of turbulent flows can be found in excellent review articles by Rogallo \& Moin (1984) and Moin \& Mahesh (1998). The first successful attempt to solve full Navier-Stokes equations in three dimensional flows was accomplished by a pseudo-spectral method for incompressible isotropic turbulence in the presence of a mean shear (Rogallo, 1981). Subsequently, DNS of wall-bounded channel flows were demonstrated with the advances in the supercomputing power (Kim et al., 1987). These simulations involved a few 
million grid points for which pseudo-spectral method for spatial discretization and semiimplicit time integration was employed. The study was the first demonstration of DNS in turbulent boundary layer flows, and provided tremendous insights into our fundamental understanding of the origin of boundary layer turbulence by unraveling the temporally and spatially resolved development of the vorticity generation. Subsequent simulations incorporated additional aspects of the specific configuration, and the database was used to extract various statistical quantities for validation and comparison with experimental measurements.

In comparison, DNS of compressible turbulent flows came out almost a decade later (Lee et al., 1991, Sarkar et al., 1991), investigating the interaction of turbulence, shock, and boundary layers. As discussed in the previous chapter, computational solution algorithm for compressible Navier-Stokes equations are made in a simple form of the method of lines solving for a system of ordinary equations, in contrast to the incompressible counterpart (Bell et al., 1989) which involves iterative solutions of the pressure Poisson equation, but at the expense of smaller time steps due to the need to resolve acoustic time scales. The pros and cons of the compressible versus low-Ma solution algorithms will continue to be debated. While the latter approach clearly has an advantage of using a larger time step (at the expense of increased computational cost per time step), the argument becomes less evident in modern DNS in which a large number of species and chemical reactions are considered, whose time scales are often comparable or even shorter than the corresponding acoustic time scales. Note that around this time, computation of simple laminar flames with detailed chemistry was being established through the framework of Chemkin (Kee et al., 1989), providing the combustion community with various flame application codes to compute one-dimensional flame structures involving tens of major and minor reactive species. However, employing the detailed chemistry into multi-dimensional DNS had to wait nearly a decade due to computational hardware limitation, and thus the first multi-dimensional simulations in this era were mainly limited to simple one-step Arrhenius chemistry model.

While not directly considering real turbulent flow fields or detailed chemistry, the first DNS of multi-dimensional flame-flow interaction was realized around the same time in late 1980s using both compressible and low-Ma approaches, in the study of flame-vortex interaction. Although this configuration does not literally qualify for the definition of DNS stated in the introduction, these were the first studies that utilized the same computational framework, while a single vortex represents an elementary unit of turbulent eddy motion interacting with the flame front. In the compressible formulation, Poinsot et al. (1991) conducted parametric studies of two-dimensional (2D) planar flames subjected to an impinging vortex at various strengths, and the results were used to provide theoretical basis to modify the turbulent premixed combustion regime diagram as shown in Figure 1. One of the main conclusions was that the laminar flamelet regime boundary defined by $\mathrm{Ka}=1$ needs to be modified if the unsteady aspects are accounted for. In other words, the smaller eddies that are likely to penetrate into the laminar flame structures also have time scales that are too short to have any meaningful effects to perturb the flames, such that the laminar flame structure remains intact. An empirical fit for the "inner cut-off" length scale, the size of turbulent eddies below which the flame is not affected, was derived. This was argued as a justification that the validity of the laminar flamelet regime may be wider than predicted in the original Borghi diagram. A similar conclusion was also drawn from theoretical (Im et al., 1995) and computational (Egolfopoulos \& Campbell, 1996) studies of laminar counterflow flames. 


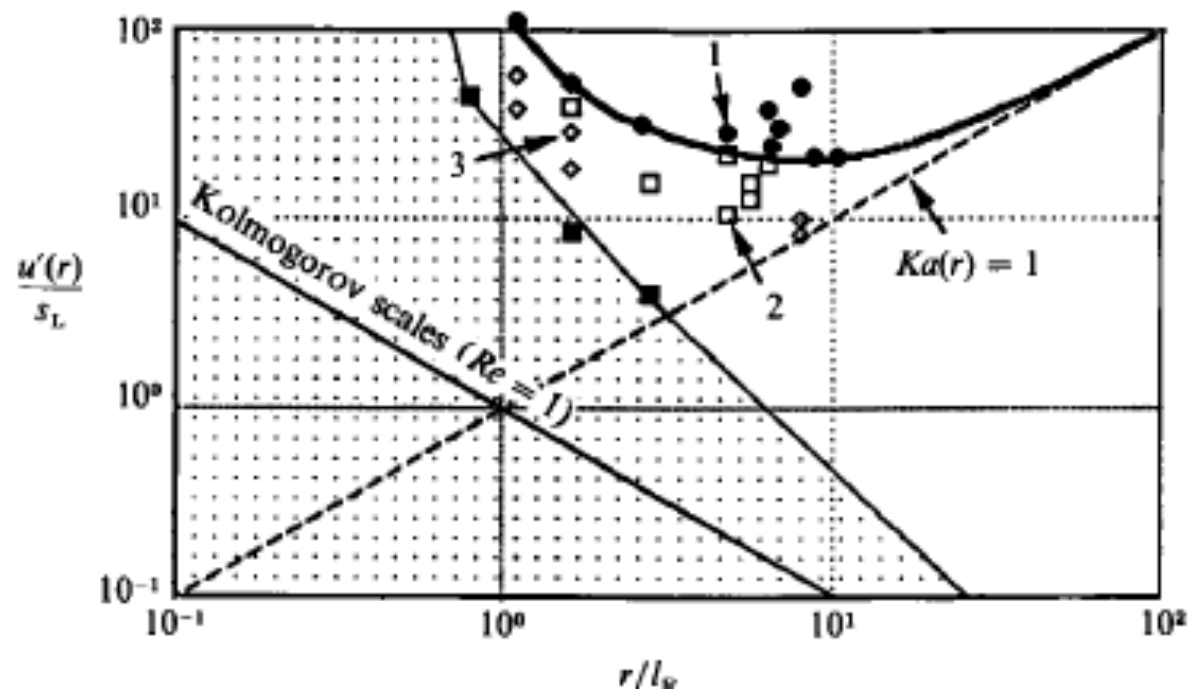

Figure 1: A revised turbulent premixed combustion regime diagram accounting for unsteadiness of turbulent eddies (taken from Poinsot et al. (1991)).

As for the first DNS using the low-Ma algorithm, Rutland and Ferziger (1991) also conducted simulations of 2D flame-vortex interaction, which quantitatively demonstrated the flame-generated vorticity through the well-known baroclinic torque involving the density and pressure gradients across the flame. As such, it was shown that the magnitude of the flamegenerated vorticity depends strongly on the amount of heat release and the resulting density variation across the flame.

Subsequent development in combustion DNS in the 1990s evolved either (a) to increase spatial complexity by incorporating three-dimensional (3D) turbulent field using simple chemistry, or (b) to increase chemical complexity by solving for detailed reactive species in 2D configurations. It took almost another decade for 3D DNS with detailed chemistry to emerge, thanks to terascale computing hardware.

\subsection{Premixed Combustion}

\section{D DNS with Simple Chemistry}

In the 3D simulations, a rectangular Cartesian domain was commonly adopted and turbulent premixed flames propagating in the streamwise direction with transverse periodic conditions with simple chemistry have been simulated, again by the low-Ma (Rutland \& Cant, 1994) and by the compressible formulation (Trouve \& Poinsot, 1994). Common in the two simulations, a steady 1D laminar flame profile with a one-step chemistry with a unity Lewis number was mapped into the 3D domain as the initial condition. A homogeneous and isotropic turbulent flow field as an instantaneous snapshot was generated in a 3D periodic cube by the Fourier transform converted from a prescribed turbulent kinetic energy spectrum (Lee et al., 1991; Baum et al., 1994). The 3D static turbulent flow field (usually at constant density and temperature) was fed at the inflow boundary surface, translated into the streamwise direction at a prescribed speed, such that the flame stays within the computational domain while the turbulence-flame interaction is fully established. As such, the turbulent kinetic energy level decays from the initial condition by the time the flow interacts with the flame. Nevertheless, a rich database was obtained that can provide valuable insights into validating many existing RANS combustion modeling approaches. 
These earlier 3D DNS studies were limited to a lower level of turbulent Reynolds number and simple chemistry, hence all the conditions were considered in the classical laminar flamelet regime in which the flame structure is only wrinkled or stretched by the turbulent eddies. Under the obvious limitations, the data field was still found to be useful in identifying the statistical distribution of flame topology. For example, by defining the flame surface as an iso-surface of a chosen scalar variable (e.g. isotherm), the flame speed relative to the local flow velocity, namely the displacement speed (Echekki \& Chen, 1998), the local flame surface curvature, and the flow strain rate can be computed by a kinematic analysis. Compiling the data on the entire flame surface have yielded the statistical distribution of the flame stretch and curvature, which are important in determining the local displacement speed according to the laminar flame theory (Williams, 1985), namely,

$$
S_{L}(\kappa)=S_{L}^{0}-\mathcal{L} \kappa
$$

where $\kappa=(1 / A) d A / d t$ is the flame stretch (Matalon, 1983) defined as the normalized Lagrangian time rate of change of the flame surface, consisting of the contributions from the flame curvature and tangential strain rate. $\mathcal{L}$ is referred to as the Markstein length, a proportionality constant that is a function of thermochemical parameters of the reactant mixture such as the Lewis number and the heat release ratio. In the laminar flamelet regime, the turbulent burning velocity is, in the first approximation, the integral of the local flame speed on the entire flame surface, and thus the distribution of the flame speed and stretch on the flame surface is important.

Another valuable contribution of the earlier 3D DNS analysis was the validation of existing turbulent combustion models. For example, Fig. 2 shows the comparison of the two DNS data discussed above, at different normalized turbulence intensities: $u^{\prime} / S_{L}^{0}=1$ for Rutland \& Cant (1994) and 10 for Trouve \& Poinsot (1994). In particular, the Favre-averaged transport equation for the reaction progress variable $\tilde{c}$ generates the flux term to be modeled as (see Chapter 5 for detailed derivation):

$$
\bar{\rho} \widetilde{u_{\imath}^{\prime \prime} c^{\prime \prime}}=-D_{t} \frac{\partial \tilde{c}}{\partial x_{i}}
$$

which is called the gradient transport model, and the Bray-Moss-Libby (BML) model:

$$
\bar{\rho} \widetilde{u_{l}^{\prime \prime} c^{\prime \prime}}=\bar{\rho} \tilde{c}(1-\tilde{c})\left(\bar{u}_{i b}-\bar{u}_{i u}\right)
$$

where $\bar{u}_{i u}$ and $\bar{u}_{i b}$ are the conditional average velocity in the unburned and burned gases, respectively. For a typical passive scalar variable, the flame propagates from $\tilde{c}=1$ to $\tilde{c}=0$ with $\bar{u}_{i b}-\bar{u}_{i u}<0$, hence $\bar{\rho} \widetilde{u_{l}^{\prime \prime} c^{\prime \prime}}>0$ according to Eq. (3). This contradicts the gradient transport model, Eq. (2), which requires the flux to be negative in order for the turbulent diffusivity, $D_{t}$, to be positive. This is the well-known "counter-gradient diffusion" problem in combustion modeling. Considering that the problem arises from the presence of the flames, it is anticipated that the effect may be attenuated as the turbulence intensity increases.

3D DNS data serve as an excellent benchmark to validate this hypothesis. The transverse periodicity of the simulation allows an approximate evaluation of the statistical averaging conditional on the mean progress variable, $\tilde{c}$, by averaging one or multiple instantaneous solution fields of the simulation. Figure 2 shows the actual turbulent flux term (LHS of Eq. (3)) and the BML term per Eq. (3) (RHS of Eq. (3)), for the two DNS data at high and low turbulence intensities. The positive values across the entire range of $\tilde{c}$ indicates counter- 
gradient diffusion (dashed lines for $\frac{u^{\prime}}{s_{L}}=1$ ), and the negative gradient diffusion is observed for $\frac{u^{\prime}}{S_{L}}=10$, confirming that the counter-gradient diffusion effect vanishes as the turbulence intensity increases.

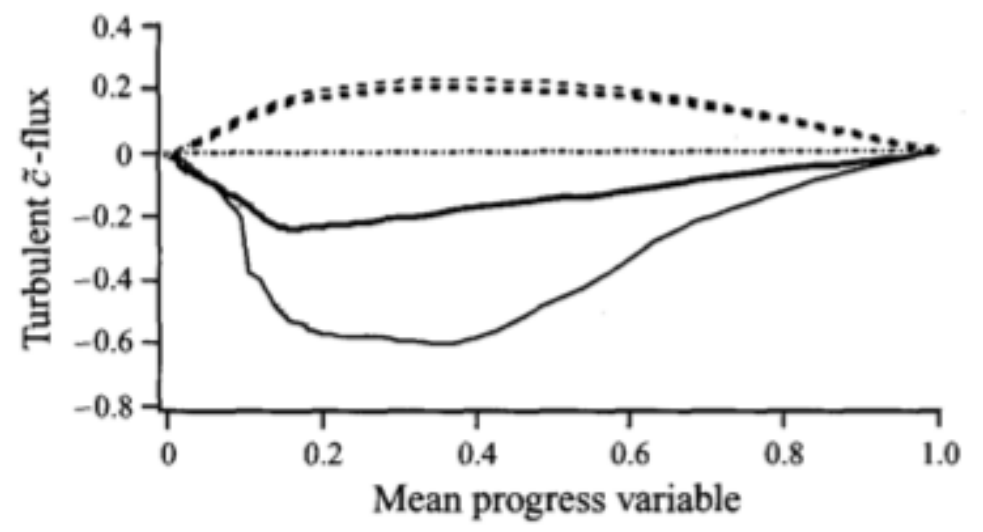

Figure 2: Comparison between the DNS data for $\frac{u^{\prime}}{s_{L}^{0}}=10$ (solid; Trouve \& Poinsot (1994)) and $\frac{u^{\prime}}{s_{L}^{0}}=1$ (dashed; Rutland \& Cant (1994)). The bold lines denote the turbulent flux terms directly computed from the DNS data, and the thin lines the corresponding BML model evaluation per Eq. (3).

3D turbulent flame simulations have since been extensively used in validation of various turbulent combustion models, an excellent summary of which can be found in the book by Poinsot \& Veynante (2005). Even today, 3D DNS with simple chemistry are being studied at higher Reynolds and Karlovitz number conditions in favor of their advantages in computational cost. One must bear in mind that the simple chemistry model is perfectly valid approximation depending on science questions under investigation.

\section{D DNS with Detailed Chemistry}

Towards mid 90s for about a decade, high fidelity DNS with full consideration of detailed chemistry emerged. Even with the simplest fuel like hydrogen/air combustion, the total number of species is increased to nine and nineteen elementary reaction steps, making 3D computations prohibitive during the gigaflops era. While some would argue that 2D turbulence is not realistic due to the absence of the critical vortex stretching mechanism, the value of 2D DNS can be justified provided that the data are used wisely. That is, it may be questionable to extract the kinetic energy spectrum and transfer which are distinct in $3 \mathrm{D}$, but 2D data provides useful information by reproducing a large number of interaction incidents between the turbulent eddies and flames. In other words, it is inappropriate to analyze the distribution of the scales of turbulent eddies and their evolution over time (which is often in reverse direction in 2D), but capturing the moment of eddy action in corrugating and disrupting the flame structure bears physical relevance. Further justification of 2D turbulence analysis has also been based on the observation of 3D homogeneous isotropic turbulent flow simulations (Ashurst et al., 1987), showing that the vortical structures are more likely cylindrical than spherical. 
2D DNS of turbulent premixed flames in a channel have been undertaken with a hydrogen-air detailed mechanism (Baum et al., 1994, Chen \& Im, 2000), with a methane-air skeletal mechanism (Echekki and Chen, 1996, Chen \& Im, 1998, Chen et al., 1998), and later with propane-air skeletal mechanism (Haworth et al., 2000; Jimenez et al., 2002). Since 2D turbulence does not evolve in a physical manner over time, most of these simulations started with superimposing a 2D slice of 3D turbulent flow onto the initial planar premixed flame, and observed the flame-turbulence interaction while the turbulence was decaying in time. Again, this was not an ideal data set to investigate turbulent kinetic energy statistics, but nonetheless was able to capture the details of the flame and eddy structures during the interaction. Figure 3 shows an example of instantaneous images of the hydrogen $\left(\mathrm{H}_{2}\right)$ consumption rates for hydrogen-air premixed flames at different mixture equivalence ratios (Chen \& Im, 2000). Consistent with the diffusive-thermal instabilities based on the laminar flame theory (Williams, 1985), it is seen that the local attenuation/quenching of the fuel consumption rate was noticeable at the trough region with negative flame curvature towards unburned gas for the fuel lean mixture (Figure 3(b)), while the opposite trend was observed for the fuel rich mixture condition (Figure 3(c)).
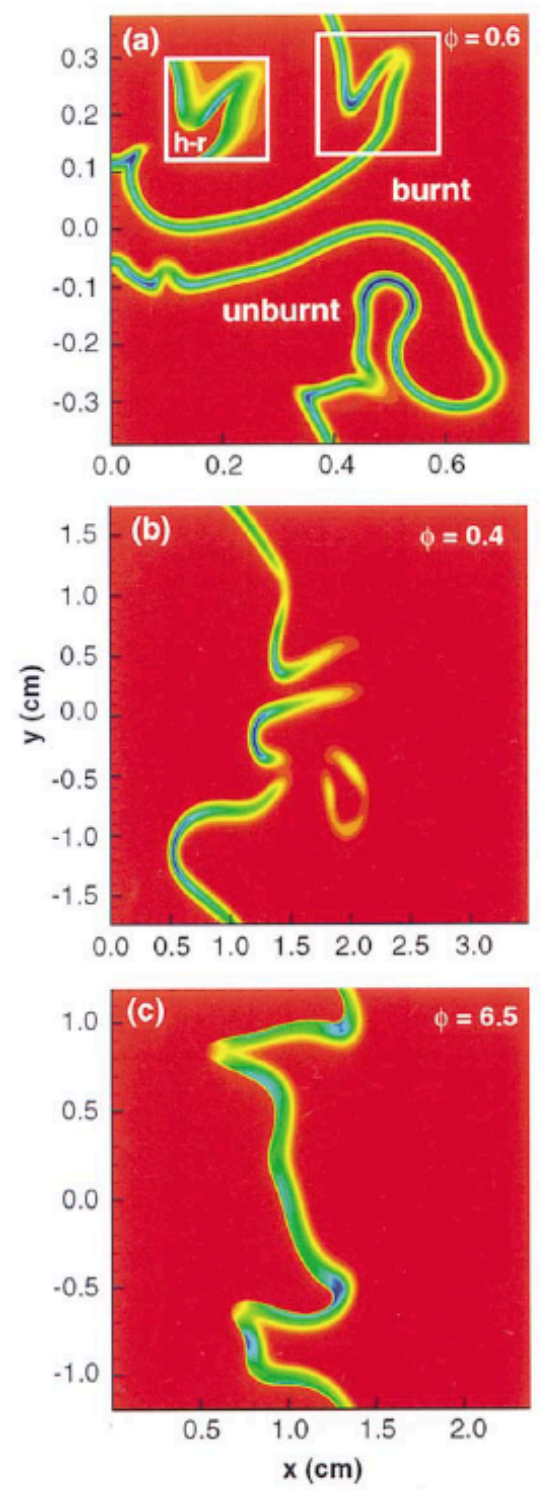
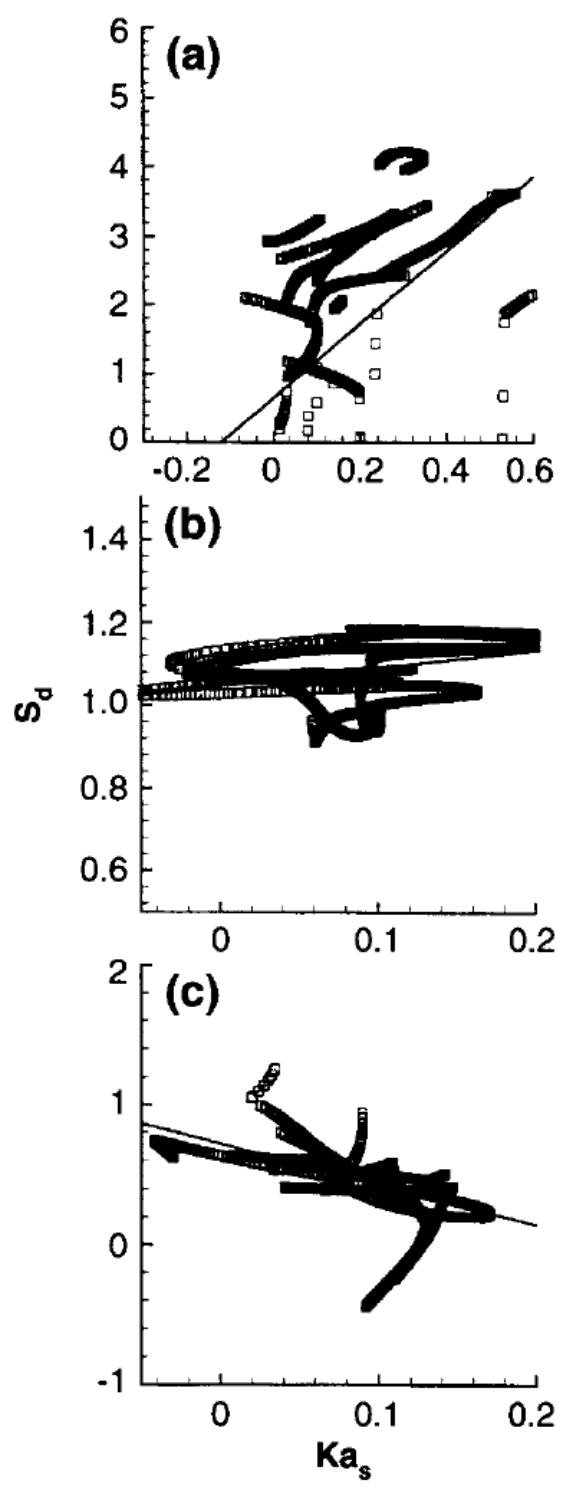
Figure 3. Iso-contours of the hydrogen consumption rate (strong in blue) in hydrogen/air premixed flames interacting with 2D turbulent flow fields, for three different equivalence ratio conditions: $\phi=$ 0.6, 0.4, and 6.5 (Chen \& Im, 2000).
Figure 4. Correlation of normalized displacement speed versus normalized tangential strain rate for the three simulation cases corresponding to Figure 3 (Chen \& Im, 2000).

Since the 2D DNS provides a large collection of different flame-eddy interaction incidents in terms of the magnitude and signs of the flame stretch and curvature, the data has been analyzed to assess the laminar flame theory as in Eq. (1), by plotting the correlation between the local flame displacement speed versus the stretch, as shown in Figure 4. While the linear correlation was found to be reasonable for moderate stretch values, some data points are seen to deviate significantly from the correlation line. These points were found to be predominantly at the flame locations where the curvature is large; it has been often the case that some extremely large stretch is more likely experienced by the curvature effect rather than the tangential straining. These types of 2D DNS data have been used to extract the the Markstein length for different hydrogen/air mixtures (Baum et al., 1994; Chen \& Im, 2000) for comparison with existing experimental measurements. While the quantitative agreement was difficult to achieve due to the large scatters in the turbulent simulation data and experimental uncertainties, a qualitative observation was noted that the Markstein length tends to be attenuated as the turbulence intensity is increases (Chen \& Im, 2000). This was attributed to the increased unsteadiness of small and fast eddies, limiting the extent to modify the flame structure.

DNS data complements experimental diagnostics by allowing access to spatially and temporally resolved information of all major and minor reactive species considered in the simulation. This offers an opportunity to determine some experimental observables by measuring alternative quantities. For example, heat release rate is an important quantity to determine combustion behavior but is difficult to measure. DNS with full consideration of detailed chemical pathways allows such alternative methods through the analysis of different chemical species and reaction signature and setting up proper correlations. Najm et al. (1998) have conducted DNS simulations of methane-air premixed flames interacting with vortices, and suggested various possible flame diagnostics in terms of cross-products of different species concentrations. For the methane-air premixed flames, the peak value of the product of $\mathrm{OH}$ and $\mathrm{CH}_{4}$ or $\mathrm{OH}$ and $\mathrm{CO}$ were found to yield excellent correlations with the heat release rate with little systematic effects due to mixture composition or flow disturbances.

\subsection{Nonpremixed Combustion and Ignition Studies}

In contrast to a long list of 2D and 3D DNS for turbulent premixed flames, studies of the nonpremixed flame counterpart are relatively scarce. This is mainly because statistically steady nonpremixed flames are difficult to establish in a similar rectangular domain. Even if the solution field is initialized by a fuel versus air mixing layer, the mixing thickness continues to grow due to molecular transport unless a mean tangential strain rate is imposed to the flame, similar to the opposed-jet flames in 1D studies. More realistic geometries like nonpremixed jet flames require a very long computational domain and were cost-prohibitive. Indeed, only a few studies are available in the literature on DNS of nonpremixed flames with growing thickness and decaying turbulence (Mahalingam et al., 1995; Bedat et al., 1999). With a one-step chemistry model, these limited studies attempted to compare the flame structure with the theoretical laminar flames in the mixture fraction space. 
For a nonpremixed combustion condition, a more commonly studied DNS configuration is ignition of a mixing layer subjected to a superimposed turbulent flow field. One of the first DNS work with simple chemistry was by Mastorakos et al. (1997), in the study of cold air facing hot air for autoignition source. The effects of the imposed turbulent flows were investigated. When the $2 \mathrm{D}$ solution field was mapped in the mixture fraction space, the "most reactive" mixture fraction, $Z_{M R}=0.1$, was identified as the preferred spot for autoignition, and the temporal evolution of the reaction progress during the ignition process was monitored. From this finding, a modeling framework based on the conditional moment closure (CMC) was proposed, emphasizing the significance of the conditionally averaged reaction rate and scalar dissipation rates near $Z_{M R}$. Subsequent studies using detailed hydrogen/air chemistry have followed (Im et al, 1998; Hilbert \& Thevenin, 2002; Hilbert et al., 2002) with consistent results. For consideration of detailed hydrogen chemistry and transport, it was found that the net ignition delay time showed a non-monotonic response to the increase in the turbulence intensity (Im et al., 1998), in that the ignition delay time slightly increases with turbulence level, and then decrease as the turbulence intensity increases further. Considering that the ignition is inherently a local event, the overall ignition behavior was found to be insensitive to the level of turbulence.

\subsection{Partially Premixed Combustion}

One of the highly active theoretical combustion research subjects at the turn of the century was the structure and dynamics of the partially premixed combustion. When a segment of nonpremixed flames becomes extinguished by an excessive local straining, as encountered at the base of the lifted jet flames, just ahead of the extinguished flame edge there is instant mixing of fuel and oxidizer, creating a tribrachial or triple flames whose edge propagates along the stoichiometric mixture fraction line, as shown in Figure 5. The bow shape of the frontal part of the flame behaves like a premixed flame front propagating onto a mixture with a transverse gradient in the equivalence ratio, and its propagation velocity (may be positive or negative) is found to be a strong function of the neighboring flow field and mixture distribution, such as the scalar dissipation rate. The specific edge flame dynamics determines whether the nonpremixed flame hole will be healed or will lead to the total extinction, and the subsequent stabilization of the lifted nonpremixed flames. While there had been a number of theoretical studies available in conceptualizing the edge flame dynamics, as summarized by Buckmaster (2002), high fidelity DNS simulations further advanced our fundamental understanding by reproducing the formation of the edge flames in realistic flow field (Domingo \& Vervisch, 1996) and their temporal evolution. Simulations of edge flames with detailed chemistry (Echekki \& Chen, 1998; Im \& Chen, 1999) have further provided detailed structure of major and minor species distributions in asymmetric edge flames, and the evolution of the flame speed as it propagates along the mixing layer was analyzed in detail. The existence of negative edge displacement speed in the presence of strong tangential straining has also been demonstrated and the transient dynamics have been investigated (Favier \& Vervisch, 2001; Im \& Chen, 2001; Yoo \& Im, 2005). These detailed simulations provided insights into the development of modeling framework for partially premixed combustion in turbulent flows (Peters, 2000). 


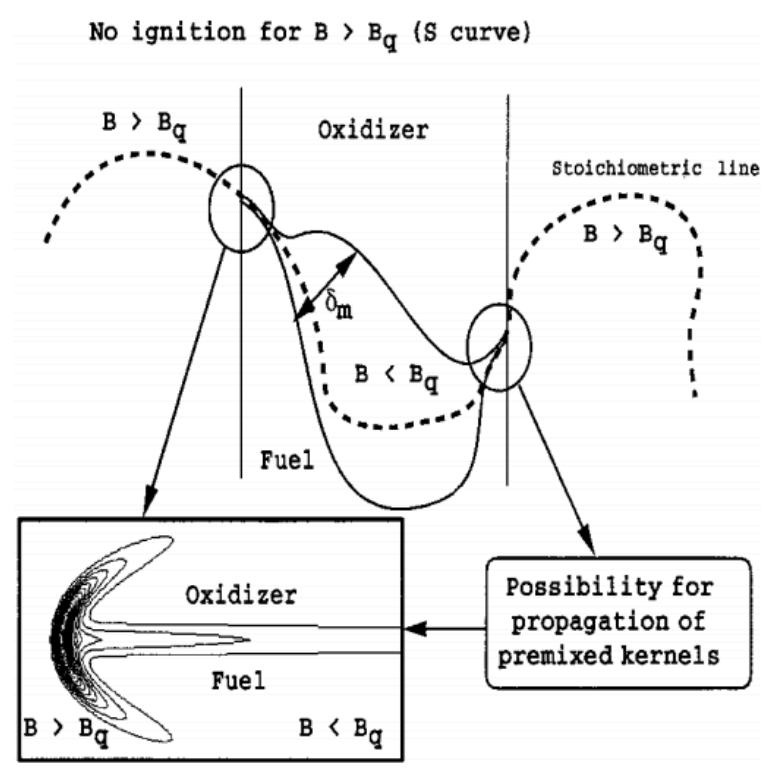

Figure 5: Schematic of nonpremixed flame quenching and the structure and mechanism of the edge flame formation (Domingo \& Vervisch, 1996).

\section{Recent Advances in DNS - Tera-, Peta-scale, and Beyond}

At the turn of the new millennium, supercomputing power at teraflops $\left(10^{12}\right.$ floating point operations per second) scale became available, thereby allowing for the first time some serious levels of 3D DNS simulations with detailed chemistry at the physical scale and parameter ranges approaching those of laboratory flames (Chen, 2011). These studies opened a new avenue in the way scientific questions are answered on turbulence-chemistry interactions and their implications in turbulent combustion modeling. This section attempts to give an overview of some notable science achievements made by large scale DNS simulations.

\subsection{Premixed Combustion}

\section{Scientific and Computational Considerations}

Turbulent burning velocity has long been a key observable quantity with practical significance, as it directly determines the performance of premixed combustion systems. A large number of review papers are available with continual updates on the progress in our fundamental understanding of the subjects (Pope, 1987; Bradley, 1992; Poinsot et al., 1995; Poinsot, 1996; Bradley, 2002; Bradley et al., 2011; Ronney, 1995; Lipatnikov et al., 2002, 2005, 2010; Driscoll, 2008). Advances in the laser diagnostics and computational capabilities enabled even deeper investigations into the important issue by analyzing spatial and temporal details of the instantaneous flame structures at unprecedented resolution and fidelity.

More recent articles give a good overview of some mainstream developments and specific computational aspects of turbulent combustion studies (Hilbert et al., 2004; Chen, 2011; Im et al., 2016). The increase in the computing power in the recent decades rapidly expanded the 
roles of DNS as an essential tool to provide insights into the intricate dynamics of the turbulence-chemistry interaction with more details of real fuel chemistry and at parametric conditions approaching those realized in the laboratory. In fact, typical spatial resolutions employed in modern DNS range from a few to tens of microns, with temporal resolution at a few to tens of nanoseconds. Thus, provided that the thermochemical properties and rate constants are accurate and the numerical schemes robust, DNS yields the detailed information about the small scale physics much better than the state-of-the-art experimental counterpart.

The latest landmark DNS cases commonly involve a few to tens of billion grid points, and with tens of reacting species with usually less than a hundred elementary reaction steps. A typical simulation case requires a few to tens of million CPU hours on the latest supercomputing hardware with the MPI (message passing interface) protocol. A caveat must be noted on the CPU hour requirements: note that temporal evolution of unsteady dynamics is critical in DNS but cannot be parallelized. Therefore, the physical time duration of the problem directly affects the total computational cost; two simulation cases at seemingly comparable physical scales and chemical complexities may take one week or one year depending on the time scales of observation. This has an important implication in the selection of the DNS cases for various combustion problems. For example, a turbulent channel flame propagation in a $1 \mathrm{~cm}^{3}$ volume with a few large eddy turnover times may require a simulation up to a few tens of milliseconds in the physical time. For the same physical scale of simulations involving auto-ignition events, the total length of the simulation time depends entirely on the overall ignition delay times, which can easily vary by more than orders of magnitude depending on the bulk temperature and pressure even for practically relevant conditions. One must be keenly aware that the CPU requirement of any types of DNS simulations can quickly become cost-prohibitive by pushing the envelope of the physical conditions of the problem under study. For this reason, there are only a few canonical computational geometries adopted for 3D DNS of turbulent premixed flames. These will be briefly reviewed in the following.

\section{Rectangular Periodic Channels}

Due to the many practical limitations discussed above, by far the most commonly employed configuration is a rectangular channel with inflow/outflows in one direction and periodic on four sides. This is an excellent reference geometry to study the basic flame-turbulence interaction to validate the theoretical predictions which are mostly based on the homogeneous and isotropic turbulence. Such an ideal turbulent flow field is generated either by using a prescribed spectrum (von Karman \& Pao) (Baum et al., 1994), mapped onto the 3D physical domain by the fast fourier transform (FFT). Alternatively, a similar homogeneous and isotropic turbulent flow field may be generated efficiently by solving an incompressible spectral solver (e.g. Rogallo, 1981). An instantaneous snapshot of the 3D flow field is then fed into the inflow rectangular surface at a prescribed inflow velocity following the Taylor's hypothesis (Taylor, 1938). The inflow mean velocity may be fixed or varied over time to ensure that the propagating flame remains inside the computational domain. This approach has an issue that turbulence intensity decays over the distance as the eddies at the inflow travels the domain to reach the flame front. To alleviate this problem, recent DNS simulations employed various types of kinetic energy forcing. The forcing may be imposed in the spectral or physical space, and it may be applied only in the upstream unburned region or throughout the entire domain. The many subtle points regarding the specifics of different turbulence forcing schemes are beyond the scope of this book, and readers are referred to relevant publications (Eswaran \& Pope, 1988; Aspden et al., 2008; Lapointe, et al., 2015). With an 
effective use of the turbulence forcing schemes, the channel DNS simulations were able to push the parametric range further into higher $\mathrm{Ka}$ and $\mathrm{Re}$ conditions.

In determining the parametric conditions for the DNS simulations, the most important consideration is the position on the turbulent combustion regime diagram, namely the Borghi diagram (Peters, 2000), where different combustion regimes are distinguished in terms of the nondimensional length scales, $\ell_{I} / \ell_{f}$, and the velocity scales, $u^{\prime} / S_{L}$. Figure 6 is a compilation of conditions covered by recent DNS simulations (Tanahashi et al., 2002; Minamoto et al., 2011; Shim et al., 2013; Aspden et al., 2011a, 2011b, 2015; Hawkes et al., 2012; Chatakonda et al., 2013; Hamlington et al., 2011, 2012; Carlsson et al., 2014, 2015; Savard and Blanquart, 2015; Savard et al., 2015; Nikolaou and Swaminathan, 2015; Minamoto et al., 2014), which needs to be updated as more new simulation data continue to be reported. A great majority of the simulations shown here employed hydrogen/air or methane/air flames with detailed chemistry, and some limited amount of studies are available with other higher hydrocarbon flames (Aspden et al., 2011c; Savard \& Blanquart, 2015; Savard et al., 2015).

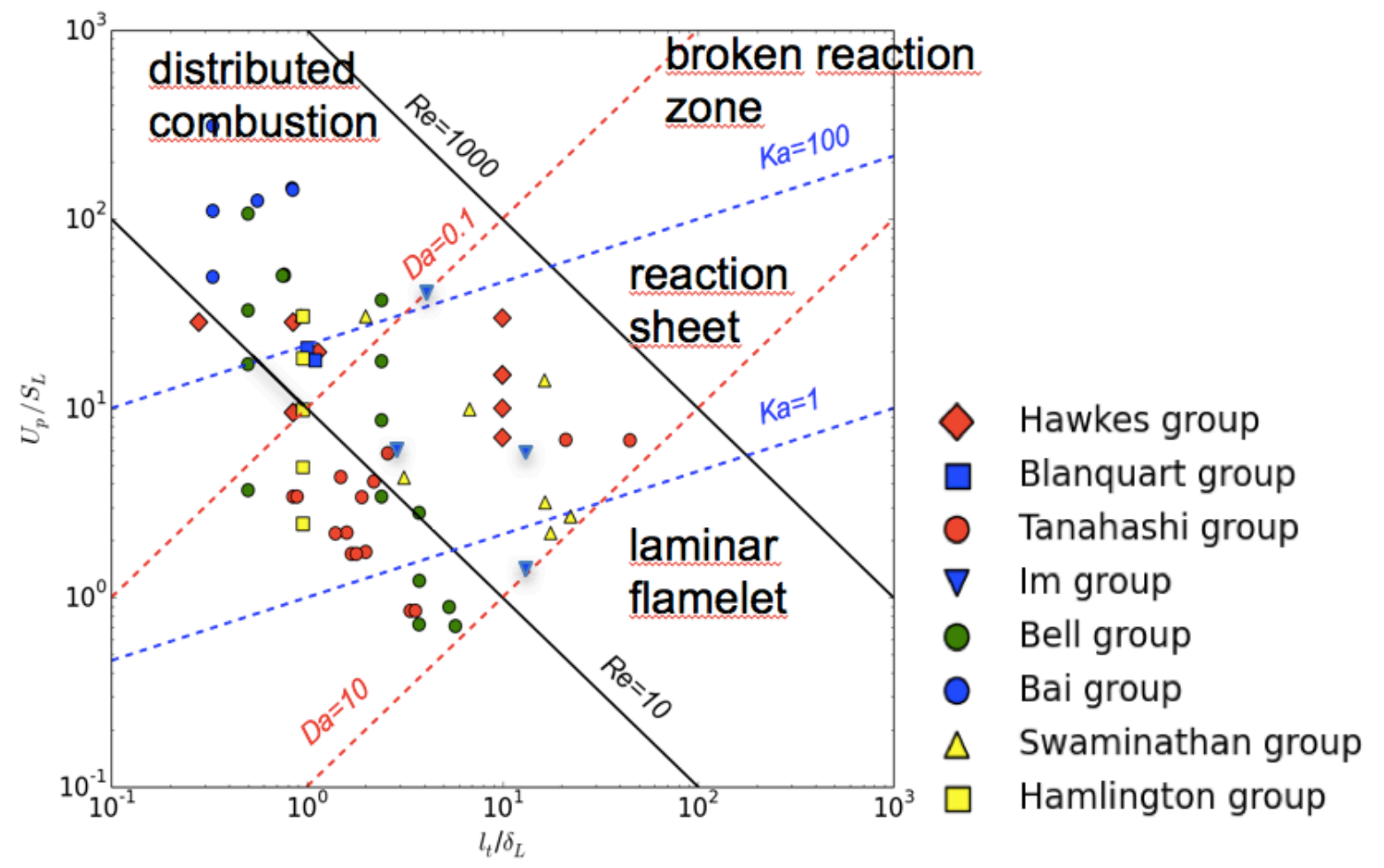

Figure 6: The Borghi diagram and the data points representing recent DNS simulation cases (figure adapted from Im et al., 2016).

The most important nondimensional parameter that determines the combustion regime is the turbulent Karlovitz number (Peters, 2000):

$$
K a=\frac{\tau_{c}}{\tau_{K}}=\left(\frac{\ell_{K}}{\ell_{f}}\right)^{2}
$$

where $\tau_{\mathrm{c}}$ and $\ell_{\mathrm{f}}$ are the flame time and length scales, respectively, and $\tau_{\mathrm{K}}$ and $\ell_{\mathrm{K}}$ denote the Kolmogorov turbulent eddy time and length scales, respectively. According to Peters (2000), the conventional laminar flamelet regime is expected for $\mathrm{Ka}<1$, the reaction sheet regime for $1<\mathrm{Ka}<100$, and broken or distributed combustion regime for $\mathrm{Ka}>100$. Figure 7 shows instantaneous solution fields for two cases at $\mathrm{Ka}=14$ and 126 based on the inflow conditions 
(Im et al., 2016). Constant temperature iso-surfaces were rendered as the flame front, with the color denoting the heat release rate intensity. As expected from the Borghi diagram, the $\mathrm{Ka}=$ 14 case shows highly wrinkled laminar flamelet structure, while the $\mathrm{Ka}=126$ case does not show distinct and contiguous flame surface, suggesting that a nearly distributed combustion regime was achieved.
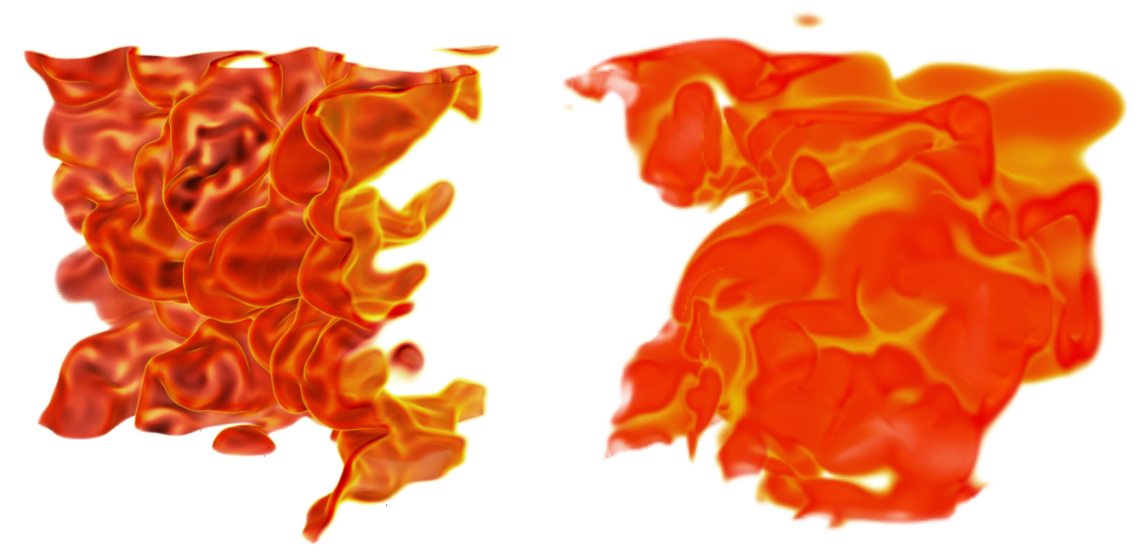

Figure 7. Instantaneous images of the heat release rate isotontours for statistically stationary turbulent premixed hydrogen-air flames at $\mathrm{Ka}=14$ (left) and 126 (right), representing the corrugated laminar flamelet and distributed combustion regimes, respectively (Im et al., 2016).

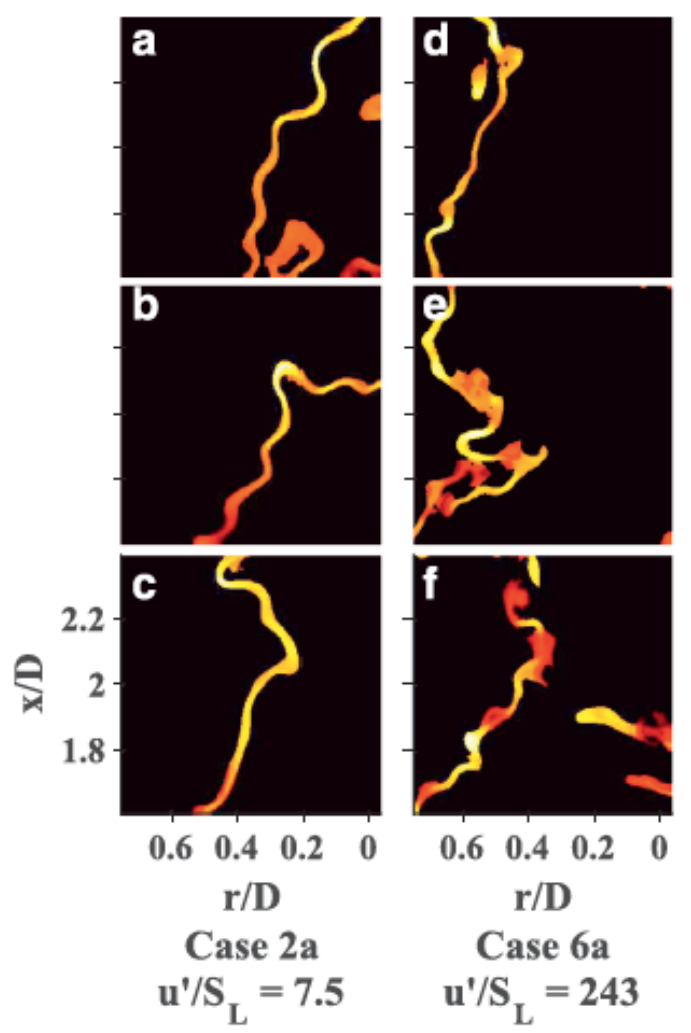

Figure 8: experimental measurements of highly turbulent methane-air premixed flames at two different conditions of turbulence intensities (Wabel et al., 2017) 
While the numerical simulations confirmed the general flame topologies consistent with the theoretical predictions, the true distributed combustion regime has yet to be experimentally observed. Figure 8 shows recent experimental results of the reaction zones denoted by the OH-LIF measurements (Wabel et al., 2017) for two cases of turbulence intensities at three different locations. While the broadening of the thermal thickness was noted, there is no clear evidence that a significant portion of the flames show distributed combustion regime, even for $\frac{u^{\prime}}{s_{L}}=243$. This is an open question today and needs to be investigated further.

The majority of the DNS results fall in the laminar flamelet regime and the 3D data were used to assess various issues investigated in the previous 3D DNS with simple chemistry or 2D DNS with detailed chemistry, in terms of the flame topology, statistical distribution of the curvature and tangential strain rate on the flame surface, the turbulent burning velocity $S_{T} / S_{L}$ vs. $u^{\prime} / S_{L}$ (Nikolaou \& Swaminathan, 2015). Most of these results were found to be by and largely consistent with the findings from the simple chemistry DNS results, with a few notable exceptions:

- For the hydrogen/air flames, the location of the main heat release, which is associated with $\mathrm{HO}_{2}$ and $\mathrm{H}_{2} \mathrm{O}_{2}$ reaction steps, is much further ahead in the lower temperature preheat zone, and thus shows a larger deviation in the flame structure compared to the reference laminar premixed flame (Tanahashi, 2002; Carlsson et al., 2014, 2015; Im et al., 2016).

- The diffusive-thermal instability mechanism is evident as expected from the laminar flame theory (most notably for lean and rich hydrogen-air flames), although the effective Lewis number of the mixture is not as obvious due to the existence of a number of radical species (such as $\mathrm{H}$ or $\mathrm{H}_{2}$ as an intermediate generated in hydrocarbon flames) whose direction of diffusion is opposite to the direction of the fuel (Chen and Im, 2000; Carlsson et al., 2015)

- The preferential diffusion effect for hydrogen or higher hydrocarbon flames arises due to the large imbalance in the molecular weight among different major and minor species. The effect, however, gradually vanishes as the turbulence intensity increases, so that the turbulent flames approaches the behavior of equi-diffusive $(\mathrm{Le}=1)$ flames (Aspden et al., 2011c; Savard et al., 2015).

- The attempt to obtain the quantitative prediction of the $S_{T} / S_{L}$ vs. $u^{\prime} / S_{L}$ curve was difficult. This is in part due to the difficulties in extensive parametric simulations for the size of expensive 3D simulations, and in part due to the relatively small domain size that limits the growth and energy transfer from large scale turbulent eddies. This is an area of active research today (O'Brien et al., 2017), with the help of the petaand exa-scale computing infrastructure that allows 3D simulations at significantly large scales.

- The effect of flames on topology of turbulent eddies and their breakdown in the enstrophy transport equations have recently been studied (Hamlington et al., 2011, 2012; Bobbit \& Blanquart, 2016; Wacks et al., 2016). The overarching conclusion is that the flame-generated turbulence resulting from the baroclinic torque mechanism is diminished as the turbulence intensity increases. Therefore, the significance of flamegenerated turbulence in determining the overall turbulent flame dynamics and propagation is still not clearly understood. 
- There are a few unique turbulent combustion conditions explored in the same rectangular geometry. Minamoto et al. (2014) studied the DNS of syngas-air flames at MILD condition, at which the mixture is significantly preheated above the ignition temperature. A kinematic analysis of the flame topology revealed a much richer spectrum of different types of flame structures, including an increasing preponderance of distributed combustion-like regimes. This finding may be an opportunity to represent the possible practical conditions where the distributed combustion regime is relevant. This is a new area of research to be further investigated.

\section{Spherically Expanding Flames}

Another relatively simple computational domain to explore turbulent premixed flame dynamics is a spherically expanding flames. This configuration is of practical interest considering many experimental studies (Abdel-Gayed et al., 1987; Liu et al., 2012; Chaudhuri et al., 2012) of turbulent flame propagation have been conducted in a spherical combustion chamber. The computational geometry can be set up as a cubic domain with uniform mesh, and a flame kernel can be place at the center to initiate flame propagation. While the computational set-up is simple, there are a number of obvious difficulties, including the initial transients, the limitations in the flame growth due to the domain size, the mean flame curvature that can bias the results and interpretations, and the difficulty in maintaining the level of turbulence intensity after the start of the simulations. The first 3D spherical DNS simulations were reported by Jenkins et al. $(2002,2006)$, and the simulations with detailed chemistry have been recently conducted (Tomboulides, 2013). Despite the limitations, the configuration is still attractive in capturing more realistic turbulent expanding flames encountered in the laboratory, and with careful analysis of the data can provide important fundamental understanding.

\section{Flame-Wall Interaction}

All combustion devices have solid walls. How the propagating flames interact with the wall and boundary layers is an important practical issue, as it is often a primary source of pollutant emission due to the flame quenching by wall heat losses. The first DNS study of turbulent premixed flames impinging on a solid wall, in the form of head-on collision, was conducted with simple chemistry and 2D geometry (Poinsot et al., 1993). With the modest scale of simulation data, the standard RANS submodels, such as the law of the wall, were refined to incorporate the effect of the flame-wall interaction. Subsequent DNS studies followed for a channel flow (Bruneaux et al., 1996; Alshaalan \& Rutland, 1998), and DNS of hydrogen-air flames impinging on inert walls and the transient history of the wall heat fluxes was investigated (Dabireau et al., 2003).

More recently, the rectangular channel flames with transverse periodicity have been modified to investigate another important practical issues as encountered in the flame flashback. The top and bottom surfaces of the computational domains are specified to be solid walls with noslip and impermeable boundary conditions. The thermal condition of the wall can be made adiabatic or with some conductive heat losses. The hydrogen-air turbulent premixed flames between two parallel walls have been investigated by Gruber et al. $(2010,2012)$. As shown in Figure 9, a notable finding from the simulation was that, due to the slow flow velocity at the wall boundary layers, which is where the leading edge of the flame front is located, the diffusive-thermal instability mechanism for lean hydrogen-air flames produces lateral flame bulges, leading to intricate three-dimensional dynamics of the flame-wall interaction. Considering that this is the critical location that governs the flame flashback conditions, 
detailed understanding of the overall flame propagation behavior as a function of various system parameters is needed.

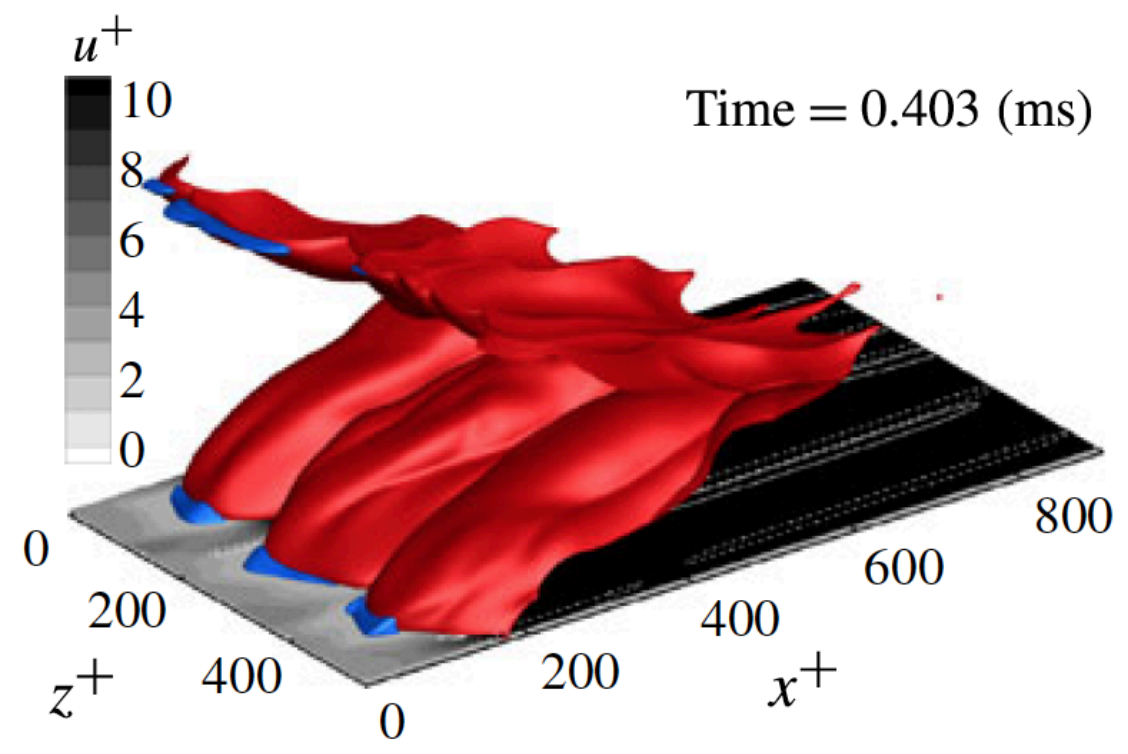

Figure 9: Premixed flame iso-surface (red) and the streamwise velocity in the viscous sublayer $\left(u^{+}\right)$in an instantaneous image of the unsteady simulation of hydrogen-air premixed flame propagation in parallel walls (Gruber et al., 2012).

\section{Temporally Evolving Shear Layer}

Hawkes and coworkers $(2012,2013)$ have conducted a series of DNS simulations of turbulent premixed flames in a unique configuration. As an attempt to achieve a high level of turbulence intensity, a 3D rectangular domain was set up such that a fuel-air mixture strip is sandwiched between the burned product gases, thus forming parallel premixed flames propagating onto each other. The center fuel strip is initially superimposed with an isotropic broadband turbulent flow field, and the entire strip is then moved at a given velocity in parallel to the flame front, generating a strong shear layer which serves as a source of turbulent kinetic energy. This configuration is often referred to as the temporally evolving jet or shear layer due to the nature of streamwise periodicity. Despite a drawback that the turbulent flows continue to change the kinetic energy level throughout the simulation, which typically increases first due to kinetic energy generation by the shear and decreases later due to the flame propagation and resulting thermal expansion, the configuration is an easy way to achieve a long time monitoring of flame-turbulence interaction without a large domain size. Three different simulation cases were analyzed for the assessment of the unresolved wrinkling factor for an a priori study of LES subgrid model (Hawkes et al., 2012), and subsequently the fractal dimension of the low Damkohler number flames was determined to be $8 / 3$ (Chatakonda et al., 2013), in contrast to the prevailing theoretical prediction of $7 / 3$ as well as a previous channel DNS study which reported a numerical value of 2.3-2.5 (Shim et al., 2011).

\section{Turbulent Jet Premixed Flames}

From the standpoint of relevance to laboratory flames, perhaps the most desirable DNS configuration is the turbulent Bunsen flame, where the fuel/air mixture stream is supplied to form the inner cylindrical or slot jet, surrounded by the external air which is quiescent. While 
the configuration is attractive in that it closely resembles the laboratory conditions, a typical length of the flames at significantly large jet velocities requires the computational domain length to be an order of magnitude larger than the other dimensions, making the computational cost drastically high. The longer domain size in the streamwise direction also implies a long flow-through time and thus a higher computational cost. Due to this consideration, existing DNS studies were all done for a slot jet configuration with periodic boundary conditions in the lateral direction with a minimal length. While it may not exactly coincide with the laboratory burner geometry, it is computationally easier for grid generation and, more importantly, statistical average quantities can be readily obtained by averaging the solution field in the lateral direction. This way the limited physical time duration for simulation can be compensated for. In distinction from the temporally evolving jet, this geometry is also referred to as the spatially evolving jet configuration.

Thus far, two research groups have produced large scale slot jet turbulent premixed flames with methane-air skeletal/reduced mechanisms (Sankaran et al., 2007; Bell et al., 2007). The main issues investigated were the effects of turbulence on the local flame structure as well as the turbulent flame brush thickness. Subsequent studies (Sankaran et al., 2015; Wang et al., 2017) included additional and larger simulation cases to explore the thin reaction zone regime, and found that the preheat zone was thickened due to the turbulence action, while the reaction zone thickness remains largely unaffected.

\section{Bluff-body Stabilized Flames}

Another configuration of practical interest is premixed flames stabilized around a solid object, often called the bluff-body. This is a typical way to achieve stable combustion in premixed combustors in gas turbines. Many engineering problems related to the bluff-body flame stabilization and associated acoustic instability issues have been extensively studied in the past decades, but DNS of such configurations are scarce and remains computational challenge, not only due to the large computational cost to capture the dimension but also the need to embed a solid object in high fidelity numerical algorithm.

Vervisch and coworkers (2004) conducted simulations of a V-flame, where the premixed flame is anchored by a numerical wire, represented by a small kernel of burned gases with a Gaussian distribution, and turbulence was generated by a separate spectral solver and fed from the inflow. After a statistically steady state was reached, the simulation data were used to investigate various flame statistics such as average progress variable or scalar fluxes.

Figure 10 shows a recent study (Kim et al., 2017) of 2D hydrogen/air premixed flames stabilized by a square bluff body in a reduced domain size of $2 \mathrm{~cm} \times 2 \mathrm{~cm}$, with the bluff body size of $1 \mathrm{~mm} \times 1 \mathrm{~mm}$. An embedded boundary method was employed to represent a solid object in a finite difference grid structure. While the physical dimension is much smaller than a realistic combustor and thus the flame condition is inherently laminar, the unsteady flame response exhibit a wide spectrum of dynamical behavior as the inflow velocity is increased, from mild fluctuations, to symmetric and asymmetric vortex shedding, and eventually forming a vortex street pattern before the total blow-out. The high fidelity simulation data provide fundamental insights into the mechanisms for flame blow-off in practical combustors. 

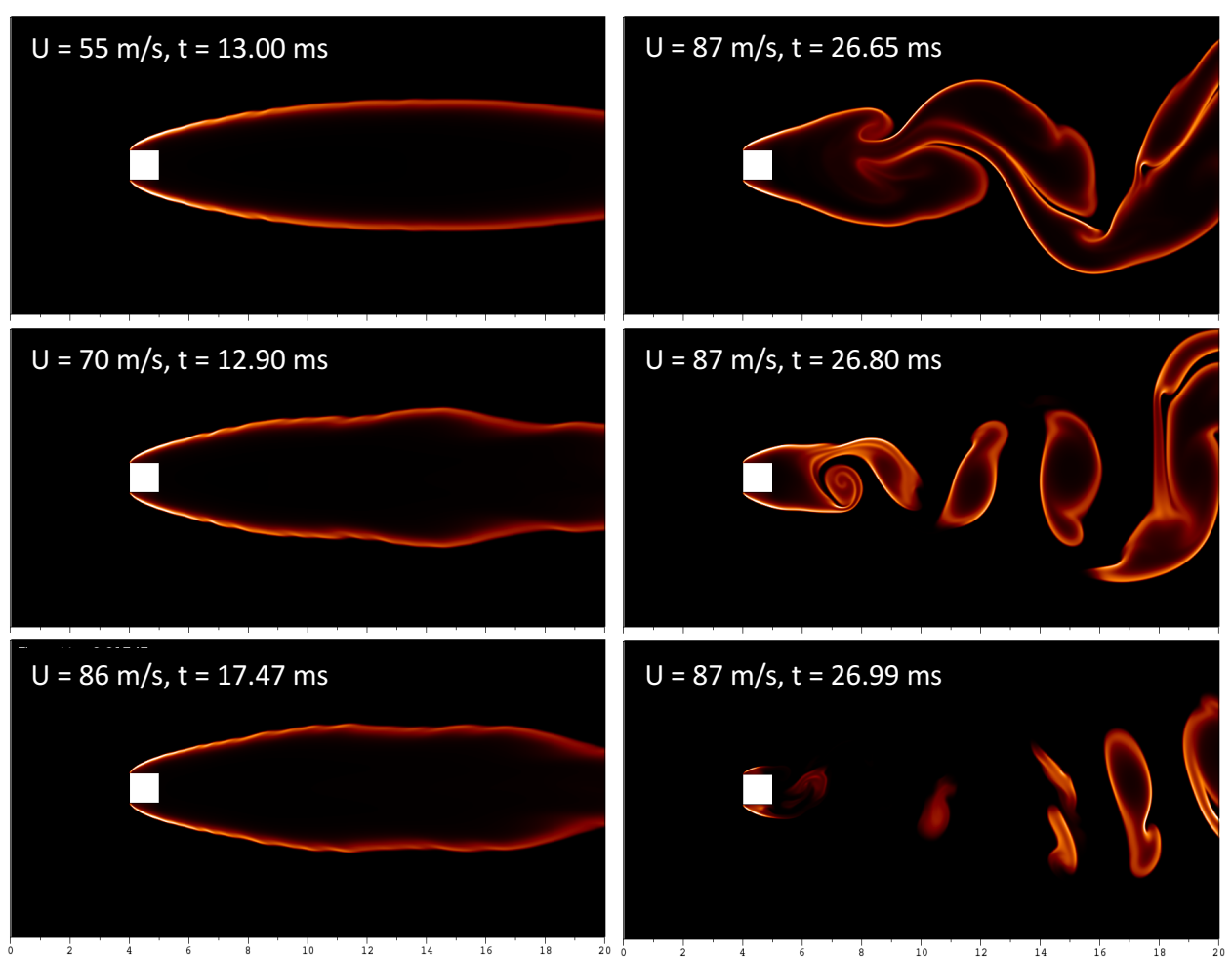

Figure 10: Instantaneous snapshots of heat release rate for hydrogen/air premixed flames anchored around a square bluff body at different inflow velocities (Kim et al., 2017).

\subsection{Nonpremixed Combustion}

Relative to the diverse simulation work on turbulent premixed combustion, fewer studies are found for turbulent nonpremixed flames. As briefly discussed in Section 2.3, any statistically steady nonpremixed flames require a finite amount of mean strain rate, either in the form of counterflow or shear layers that would occur in jet flames. Either way, the computational domain needs to capture a large physical scale that is characteristic of the burner system, such as the distance between the nozzle or the jet diameter. Therefore, the usual dimension required for the simulation is much larger than a few integral scale eddies, which is a usual practice in the channel premixed flames, making a typical turbulent nonpremixed flame simulation a larger computational challenge. This is why the 3D DNS of nonpremixed flames with detailed chemistry have been conducted some years later than the premixed counterpart. With the advances in HPC hardware, however, simulations at remarkably large scales are being conducted and reported today.

Some canonical configurations for turbulent nonpremixed flame study can be found in the Turbulent Nonpremixed Flame (TNF) Workshop (http://www.sandia.gov/TNF), a community research forum launched in 1996 with the leadership of Sandia National Laboratories. The experimental data archives show that the predominant geometry is the jet flame configuration, with or without the pilot depending on the level of difficulties in stabilizing the flames. There are some experimental set-up for turbulent counterflow nonpremixed flames (Coppola et al., 2009) using elaborate turbulence generation mechanism at the nozzle inlet. However, DNS at this scale is still expensive and no 3D simulations of this system have been reported to date. 


\section{Turbulent Jet Flames}

Despite the computational challenge, the first landmark 3D DNS of turbulent nonpremixed flames with detailed hydrogen/air chemistry was conducted by Mizobuchi et al. (2002, 2005). A hydrogen jet was injected from a cylindrical nozzle at a diameter of $2 \mathrm{~mm}$, at a velocity of $660 \mathrm{~m} / \mathrm{s}$ into the surrounding quiescent air, yielding the Reynolds number based on the nozzle diameter at 13,600 . Due to the high jet velocity, the nonpremixed flame is lifted from the burner, such that the flame stabilization mechanism and partial premixing of the reactants in the flame base region were extensively investigated. These studies are also highly cited for the assessment of the Takeno Index, defined as the product of the local fuel and oxidizer gradients, such that the positive and negative values of the index indicate the combustion mode being premixed and nonpremixed, respectively. Figure 11 shows a snapshot image of the lifted flame, with the premixed and nonpremixed combustion regimes color-coded.

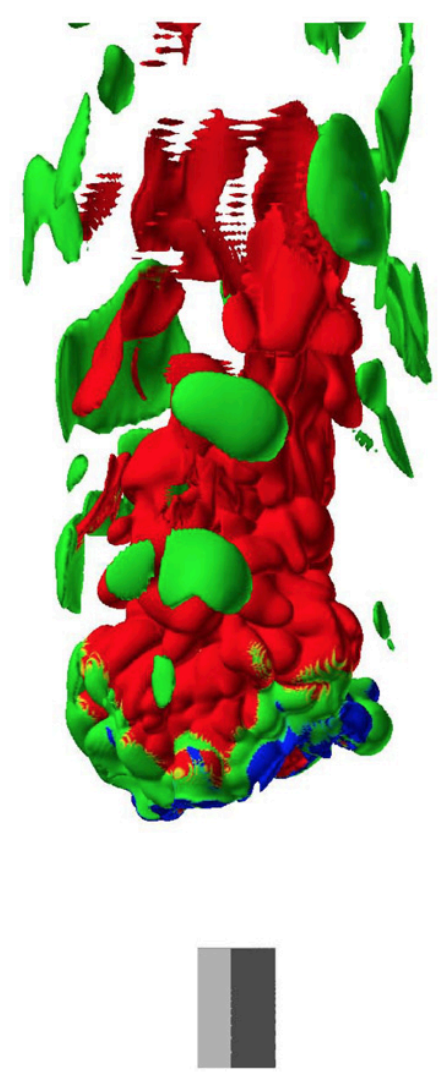

Figure 11: Global structure of the hydrogen jet lifted flame. Surface color shows the combustion mode: red (rich premixed), blue (lean premixed), and green (nonpremixed) (Mizobuchi, 2005).

Shortly after, the Sandia group also conducted a slot-jet lifted nonpremixed flames with preheated coflow air for hydrogen-air (Lu et al., 2010; Yoo et al., 2011) and ethylene-air (Yoo et al., 2011) flames, at Reynolds numbers comparable to the work by Mizobuchi et al. (2002, 2005). A rectangular slot jet of a width $2 \mathrm{~mm}$ and the jet velocity of $204 \mathrm{~m} / \mathrm{s}$ was used, resulting in the Reynolds number of 10,000 based on the nozzle width, comparable to the study by Mizobuchi et al. $(2002,2005)$. Main differences were that the air coflow velocity 
was imposed at $20 \mathrm{~m} / \mathrm{s}$, and highly preheated to $1550 \mathrm{~K}$. The coflow preheating was a pragmatic computational choice to stabilize the flame, but it also shifted the focus of the scientific study as the mechanism for the flame stabilization at the lifted base was changed to auto-ignition of the preheated reactants rather than the conventional edge flame propagation. The auto-ignitive stabilization behavior was manifested by the saw-toothed shape of the flame base trajectory in time, where the slow downstream movement was followed by a rapid upstream movement.

\section{Temporally Evolving Shear Layer}

These simulations are marked as the largest scale HPC simulations of turbulent combustion DNS to date, while a few other simulations are being presented in technical conferences. Considering the large computational cost, as in the premixed flame simulations, reduced size nonpremixed combustion DNS were also conducted in the temporally evolving shear layer configuration. Hawkes et al. (2009) conducted 3D DNS of temporally evolving shear layer, similar to that discussed in Section 3.1 (Hawkes et al., 2012), except that the inner layer was a fuel strip and the outer layers were oxidizer stream. The parallel shear flow created by the imposed lateral velocity field yielded a significantly larger level of the scalar dissipation rates on the spatially periodic dual nonpremixed flame surfaces, thereby allowing an excellent database to analyze the true $3 \mathrm{D}$ turbulence effects on the propability density function (pdf) statistics of the scalar dissipation rate on the flame surface, which is an important quantity for accurate combustion submodels for LES and RANS simulations. A new theoretical relationship was developed for the joint pdf of the scalar dissipation rate and other scalar quantities. The same computational setting was also used to study the dynamics of the sooting flames with skeletal ethylene-air chemistry and semi-empirical soot models in 2D and 3D configurations (Lignell et al., 2007, 2008).

\section{Turbulent Counterflow Flames}

Despite the advantages of compact configurations, 3D DNS of turbulent counterflow flames, either premixed or nonpremixed, have not been conducted at full scale for high Reynolds number conditions. 2D DNS of counterflow nonpremixed flames have been conducted for the edge flame dynamics for hydrogen-air flames (Yoo \& Im, 2005), 2D turbulent sooting flames with simple ethylene and soot chemistry (Yoo \& Im, 2007), and 2D turbulent ethylene flames with a skeletal mechanism (Arias et al., 2011) which also added a Lagrangian droplet transport to investigate the flame extinction by fine water mist. A 3D laminar counterflow nonpremixed hydrogen-air flame with detailed chemistry was conducted by Lee et al. (2010), investigating the effect of $\mathrm{NO}$ on extinction and re-ignition induced by vortex injection, in comparison with experimental data. The re-ignition is characterized by the positive edge flame propagation after the quenching of the flame center. The effects of NO concentration and the vortex strength on the flame recovery were investigated.

\section{Jet in Cross-flow}

The jet in cross-flow (JICF) configuration is highly relevant in high speed vehicles. The fuel jet injected normal to the air stream serves as a bluff-body for the upcoming cross-flows, so that the subsequent flame development and stabilization is facilitated by the vortices generated in the wake of the flow behind the fuel jet. While there is a long history of studying the hydrodynamic aspects - the vortex structure, breakdown, and recirculation - realistic 3D simulations of reacting JICF is rare. Grout et al. (2011) conducted a large scale 3D DNS of JICF with hydrogen-air detailed chemistry. Figure 12 shows an instantaneous image of the 
iso-surfaces of various scalar quantities. For the physical dimension of approximately $8 \mathrm{~cm}^{3}$, 1.6 billion grid points were used and the simulation was run on Cray XT5 for 5M cpu hours. The realistic 3D solution field was examined in detail to provide fundamental understanding of the flame stabilization mechanism downstream of the transverse fuel jet. It was found that the coherent vortical structures originating from the jet shear layer interaction have a strong effect in determining the upstream portion of the flames.

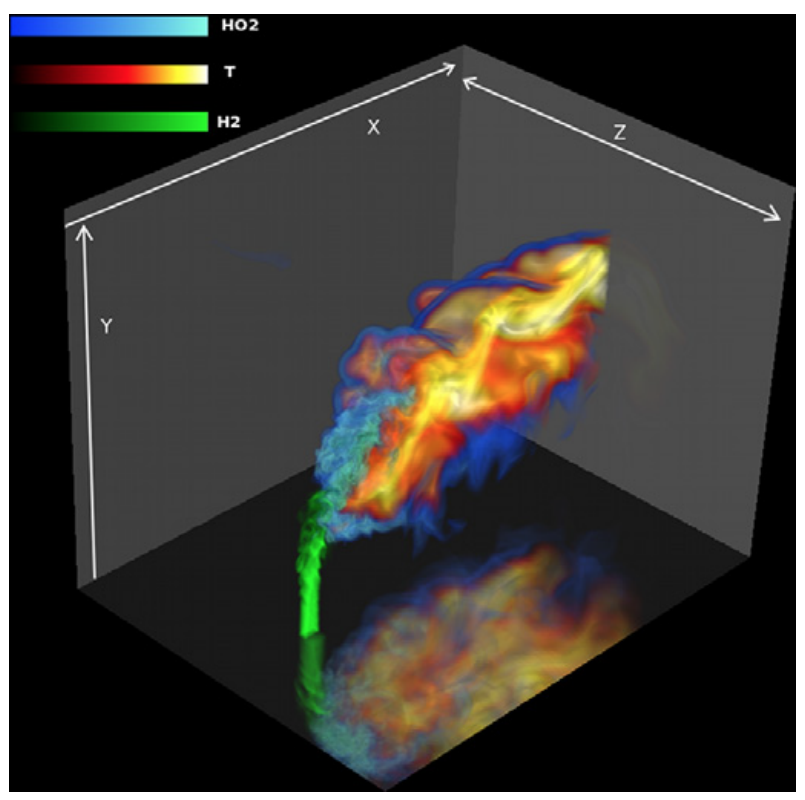

Figure 12: Volume rendering of temperature, $\mathrm{HO}_{2}$, and $\mathrm{H}_{2}$ concentrations around a nitrogendiluted hydrogen jet in a heated turbulent air crossflow (Grout et al., 2011).

\section{Future Research Opportunities in Modeling and Science}

This chapter attempted to provide a historical review of the advances in combustion DNS in both physical scale as well as the complexities of problems investigated. The past decade has seen the role of DNS from an extended reduced model to validate a smaller subset of combustion problems to a serious research tool that can directly complement or even guide laboratory experiments. It is hoped that the comparison of Sections 2 and 3 in this chapter would highlight such a dramatic progress in computational combustion research.

The HPC hardware will continue to advance, still keeping up with the Moore's law, such that the academic community worldwide are preparing for the exa-scale era. It is anticipated that the next decade will lead us into even more impressive breakthroughs in the way combustion modeling can be utilized in answering both long-standing fundamental questions as well as overcoming real life engineering design challenges for future combustion systems, which must explore vastly different design and fuel diversity in order to keep up with emerging alternative power systems in terms of energy efficiency and environmental impact. The advances in these alternative transportation and stationary power generation systems in recent times have been truly remarkable. It is obvious that combustion of conventional and alternative fuels will continue to play a key role in the next decades and beyond in favor of its energy density and existing supply infrastructure. Combustion research will remain viable for the future generation, and advanced predictive computational tools will play a vital role in 
achieving the goal.

For DNS to utilize the future exa-scale architecture successfully, there are a number of research challenges in both computational as well as physical modeling capabilities. It is important to keep in mind that the fidelity of simulations is satisfied only if the underlying physical and chemical submodels are correct and reliable at the thermochemical conditions under study, and if the embedded numerical algorithms and schemes are stable and accurate with sufficient spatial and temporal resolution. If any one of these factors is not adequate, the simulation results cannot be trusted. Another important challenge is that the specific problem configuration and physical conditions for the large scale simulations need to be carefully chosen to address the most critical fundamental issues that will have strongest impact in future combustion systems. In the following, the research challenges in both aspects towards successful exascale DNS will be discussed and summarized.

\subsection{New Models and Computational Capabilities}

\section{Physical Models}

As the computational power increases, DNS will be able to enhance the fidelity by adding more details of the underlying physical and chemical submodels. Provided that the physics models are compatible with the computational solution algorithms, the enhanced submodels will increase the realism and quantitative prediction of the simulation results. In general, the submodels must be carefully validated under controlled parametric conditions against experimental data or with a more detailed model prediction in lower-dimensional conditions for efficient computation prior to their implementation into the $3 \mathrm{D}$ production runs.

First, the detailed chemical reaction mechanism must be able to predict the needed observables (e.g. ignition delay times, flame speed, maximum temperature) accurately over the thermodynamic conditions under study. Ideally, the detailed kinetic mechanism should include all major and minor reactive species and relevant chemical pathways with accurate rate constants. However, this exercise becomes quickly unmanageable for practical fuels with a large and complex molecular structures. Even if the rate constants for each elementary reaction steps can be accurately determined, such detailed mechanisms would become too big to be employed in 3D DNS due to the large number of reactive scalars and reaction source term evaluations. Furthermore, the wide range of characteristic time scales among various reactions introduces temporal stiffness which will significantly increase the computational cost. Therefore, the mechanism development should be done judiciously by retaining the minimal number of species and reactions, while ensuring that there is no unphysical short time scales. As such, today's kinetic mechanism development for real fuels has become a large collaborative effort among the experts in quantum chemistry, experiments and laser diagnostics, and computational method for optimization and dynamical analysis toward the development of the minimal reaction mechanism. For each species considered, it is also equally important to determine the relevant transport properties in the multi-component system.

Quantitative prediction of particulate formation (soot or other combustion-generated particles) needs further advancement in the ability to determine not only the total mass and number of the particulates, but the particle size distribution which is critical in meeting various environmental regulations. Today's state of the art is higher order hybrid method of moments (Mueller et al., 2009; Salenbauch et al., 2017), for which a variety of refinements are available. Sectional methods dealing with the number density variable for each of a finite 
number of size classes have been considered a more advanced detailed approach in the aerosol modeling community, but has been computationally prohibitive for today's combustion DNS applications. With the exascale computing hardware, however, it is foreseeable that the sectional approach becomes a standard practice in soot prediction in 3D DNS. Note also that accurate soot prediction is also contingent on reliable heterogeneous kinetic mechanisms to describe the soot nucleation, growth/agglomeration, and oxidation processes.

Combustion product gases are commonly at higher temperature and a number of gaseous species are highly absorptive and emissive in thermal radiation. Furthermore, a significant level of soot production will further participate in the radiative heat transfer process. Therefore, reliable radiative heat transfer models for relevant combustion conditions are necessary. Today's DNS largely neglects the detailed radiation effects by resorting to gray gas assumption (in the spectral space) and optically thin gas approximation (in the physical space). Some DNS studies incorporated optically thick gas conditions and solve radiative transfer equations (RTE). Stochastic photon Monte-Carlo method (Modest, 2013) is being explored as a viable method that can incorporate spectral resolution of the radiative properties. To move beyond the gray approximation, at the 3D DNS level some scaled or correlated approaches such as full spectrum correlated-k (FSCK) or scaled-k (FSSK) methods are available in the literature (Modest, 2013).

Many real combustion devices deal with direct injection of liquid fuels into the combustion chamber. Depending on the injection timing, the combustion process may become extremely complex with full interactions among spray break-up, evaporation, mixing, and combustion in the surrounding air flows. The liquid spray itself contains a wide spectrum of the droplet size distribution with complex secondary break-up, collision, and agglomeration, and interaction with the solid walls. DNS to resolve the details of such multiphase flows is an extremely challenging task. A common practice in high fidelity simulations is to use the Lagrangian particle-in-cell method (Mashayek, 1998) for far field spray dynamics, and the Eulerian volume-of-fluid method for near field spray close to or inside the nozzle (Desjardins et al., 2008; Lebas et al., 2009). Full scale Eulerian spray DNS for the entire injector throughout the combustion process has yet to be demonstrated and more work needs to be done in order to accomplish this goal efficiently.

Modern combustion devices are pushing the operating conditions towards higher pressure for efficiency enhancement. For internal combustion engines, some engine cycle concepts demand combustion at 300 bar conditions. At such pressure conditions, many existing kinetic mechanisms and transport properties no longer work. Furthermore, the multiphase flow reaches supercritical conditions, and the entire interface dynamics and multi-phase mixing process becomes totally different. DNS of supercritical combustion is still a wide open area and much work is needed.

\section{Computational Capabilities}

The exa-scale supercomputers will further extend the realizable range of physical parameters to close the gap between the simulations and practical systems. To successfully utilize the new HPC hardware, however, extensive modifications of the software program and the solution algorithms are needed. It is projected that the upcoming leadership computing hardware (e.g. https://www.olcf.ornl.gov/summit/) will likely utilize a large fraction of the system equipped with multi-core accelerators, such as graphical processing units (GPU) or Intel Xeon Phi, demanding the DNS code to be optimized for the hybrid MPI $+\mathbf{X}$ 
architecture, where $\mathrm{X}$ refers to various types of multi-core accelerators, in order to achieve the maximum scalability as well as per-core speed-up.

While the computational power increases rapidly, permanent disk and archival tape storage are not expected to grow proportionately. Even with the state-of-the-art visualization and analysis tools, common analysis tasks require substantial computing resources. Consequently, the exascale era will also demand innovations in the use of large datasets for scientific discovery. It is thus expected that such discoveries will be made through in-situ/in-transit analytics (Bennett et al., 2012). That is, simulations have to be instrumented for data reduction and feature extraction to selectively reduce the volume of data that needs to be processed immediately and/or sent to archival disks and tapes. Most of today's visualization and analysis tools and workflows only process the raw data through the storage, and are not capable of such efficient data-mining and analysis.

Furthermore, one of the common difficulties in data extraction and analysis of the massive DNS data is that the parameters of the analysis themselves have uncertainties. For example, for highly turbulent premixed flames, it is unclear which scalar variable at what iso-contour value serves as the best metric to determine the overall burning velocity. As such, the current practice of analyzing the DNS data is extremely time-consuming with a large number of trials and errors. The definitions of geometrical features, statistical measures and correlations have an associated uncertainty that makes the analysis process iterative and highly dependent on the availability of massive storage capacity. As a practical implementation, the initial phases of data analysis are often highly exploratory with several subsets of data through reduction that provides the most useful information with a high degree of confidence. Performing such analysis in-situ will remain a major challenge, and require a new paradigm in data management infrastructure combined with real-time data streaming and post-processing (Pascucci et al., 2012).

Landmark DNS simulations at maximum achievable scales and parameter ranges have been attempted within the reach of available computing power. While these simulations provided tremendous insights into understanding of underlying physical mechanisms of critical phenomena under consideration, such simulations can only be undertaken for a few realizations at limited parametric conditions. Therefore, they are not well suited for systematic statistical analysis. To this end, another important role of DNS is to create a large ensemble of well-defined canonical DNS simulations. Exa-scale HPC will enable hundreds to thousands of peta-scale ensemble simulations for statistically converged data analysis over a wide range of parametric conditions along with quantifiable uncertainties.

The ability to perform a large number of simulations comes with the associated difficulties in choosing the most effective way to conduct the computational parametric studies, including the massive data management and interrogation. Therefore, there is a strong demand in automation and machine learning in the selection and prioritization of cases, their scheduling and subsequent data management. Such optimal learning techniques are employed in many other fields such as meteorology, biology, drug discovery, and combustion kinetics (Huan \& Marzouk, 2013). The optimal learning techniques start with an initial belief on the output characteristics of the simulations. The aforementioned in-situ and in-transit analysis capability allows a comparative visualization to guide the selection of the next simulation case. To this end, uncertainty quantification (UQ) (le Maitre \& Knio, 2010) plays a crucial role in the decision-making process through global analysis, parameter inference, model calibration, and Bayesian optimal design techniques. 


\subsection{Research Questions}

With the best DNS capabilities in the exa-scale era with the state-of-the-art data analytics, time is ripe to tackle some important science and engineering questions for future combustion systems. Here is a list of research topics that are currently being investigated as critical issues for modern combustion systems. As discussed before, many of the research questions are closely tied to the fact that modern combustion systems are being designed at extreme thermodynamic and physical conditions that have not been explored in the past. The following list is by no means comprehensive, and is only intended to spawn many more new ideas.

- What are the prevailing combustion characteristics at strongly turbulent conditions, represented by high $\mathrm{Ka}, \mathrm{Re}$, and $\mathrm{Da}$ ? Does the postulated distributed combustion regime really exist in practical engines?

- What are the distinct characteristics of turbulent combustion at higher pressure? That is, provided that the basic responses of chemistry and transport processes to pressure are well understood, can the turbulent combustion behavior be predicted as an extension of our existing knowledge, or are there a significant nonlinear effect associated with turbulence-flame interaction?

- What are the most critical features (e.g. phase relation, transport properties) that need to be captured for accurate prediction of multi-phase combustion at supercritical conditions? What are the proper predictive simulation strategies?

- Is the low temperature chemistry, also referred to as the cool flame phenomena, important in high pressure and high Re turbulent combustion? What is its most significant impact on the combustion performance?

- What are the distinct characteristics in pollutant formation (NOx and soot) at such extreme conditions? What are the adequate strategies to mitigate the pollutant emissions?

- How can we develop a unified combustion closure models that are applicable for mixed mode (both premixed and nonpremixed) turbulent combustion?

\section{Acknowledgments}

The work presented in this study was sponsored by King Abdullah University of Science and Technology (KAUST).

\section{References}

Abdel-Gayed, R.G. Bradley, D., and Lawes, M. 1987. Turbulent burning velocities: a general correlation in terms of straining rates. Proc. R. Soc. London A, 1847, 389-413.

Alshaalan, T.M. and Rutland, C.J. 1998. Turbulence, scalar transport, and reaction rates in flame-wall interaction. Proc. Comb. Inst., 27, 793-799. 
Arias, P. G., Im, H. G., Narayanan, P., and Trouvé, A., 2011. A Computational Study of Nonpremixed Flame Extinction by Water Spray. Proceedings of the Combustion Institute, 33, 2591-2597.

Ashurst, W. T., Kerstein, A. R., Kerr, R. M. \& Gibson, C. H. 1987. Alignment of vorticity and scalar gradient with strain in simulated Navier-Stokes turbulence. Phys. Fluids 30, 2343-2353.

Aspden, A.J., Bell, J.B., Day, M.S., Woosley, S.E., Zingale, M. 2008. Turbulence-flame interactions in type Ia supernovae. The Astrophysical Journal , 689 (2).

Aspden, A.J., Day, M.S., Bell, J.B. 2011a. Characterization of low Lewis number flames. Proc. Combust. Inst., 33, 1463-1471.

Aspden, A.J., Day, M.S., Bell, J.B. 2011b. Turbulence-flame interactions in lean premixed hydrogen: transition to the distributed burning regime. J. Fluid Mech., 680, 287-320.

Aspden, A.J., Day, M.S., Bell, J.B. 2011c. Lewis number effects in distributed flames. Proc. Combust. Inst. 33, 1473-1480.

Aspden, A.J., Day, M.S., and Bell, J.B. 2015. Turbulence-chemistry interaction in lean premixed hydrogen combustion. Proc. Combust. Inst., 35, 1321-1329.

Baum, M., Poinsot, T., Haworth, D. \& Darabiha, N. 1994 Using Direct Numerical Simulations to study $\mathrm{H}_{2} / \mathrm{O}_{2} / \mathrm{N}_{2}$ flames with complex chemistry in turbulent flows. J. Fluid Mech. 281, 1-32.

Bedat, B., Egolfopoulos, F. \& Poinsot, T. 1999. Direct numerical simulation of heat release and NOx formation in turbulent non premixed flames. Combust. Flame 119, 69-83.

Bell, J.,B., Collela, P., Glaz, H.M. 1989. A second-order projection method for the incompressible Navier-Stokes equations. J. Comp. Phy. 85, 257-283.

Bell, J.B., Day, M.S., Grcar, J.F., Lijewski, M.J., Driscoll, J.F., Filatyev, S.A. 2007. Numerical simulation of a laboratory-scale turbulent slot flame. Proc. Comb. Inst. 27, 1299-1307.

Bennett, J. C.; Abbasi, H.; Bremer, P.-T.; Grout, R.; Gyulassy, A.; Jin, T.; Klasky, S.; Kolla, H.; Parashar, M., Pascucci, V.; Pebay, P.; Thompson, D.; Yu, H.; Zhang, F.; Chen, J. H. Combining in-situ and in-transit processing to enable extreme-scale scientific analysis. Proceedings of the International Conference on High Performance Computing, Networking, Storage and Analysis, SC '12, pages 49:1-49:9, Los Alamitos, CA, USA, 2012. IEEE Computer Society Press.

Bobbitt, B., Blanquart, G. 2016. Vorticity isotropy in high Karlovitz number premixed flames, Physics of Fluids 28, 105101.

Bradley, D. 1992. How fast can we burn? Proc. Combust. Inst., 24, 247-262.

Bradley, D. 2002. Problems of predicting turbulent burning rates. Comb. Theory Modell., 6(2) 361-382.

Bradley, D., Lawes, M., and Mansour, M.S. 2011. The problems of the turbulent burning velocity. Flow Turbulence Combust., 87, 191-204.

Bruneaux, G., Akselvoll, K., Poinsot, T., Ferziger, J.H. 1996. Flame-wall interaction simulation in a turbulent channel flow. Comb. Flame, 107, 27-44.

Buckmaster, J. Edge flames. 2002. Prog. Energy Comb. Sci. 28, 435-475.

Carlsson, H. Yu, R., and Bai, X.S. 2014. Direct numerical simulation of lean premixed CH4/air and H2/air flames at high Karlovitz numbers. Int. J. Hydrogen Energy, 39, 20216-20232.

Carlsson, H. Yu, R., and Bai, X.S. 2015. Flame structure analysis for categorization of lean premixed CH4/air and H2/air flames at high Karlovitz numbers: direct numerical simulation studies. Proc. Combust. Inst., 35, 1425-1432. 
Chatakonda, O. Hawkes, E.R., Aspden, A.J., Kerstein, A.R., Kolla, H., and Chen, J.H. 2013. On the fractal characteristics of low Damköhler number flames. Combust. Flame, 160 2422-2433.

Chaudhuri, S., Wu, F., Zhu, D., and Law, C.K. 2012. Flame Speed and Self-Similar Propagation of Expanding Turbulent Premixed Flames. Phys. Rev. Letters, 108, 044503.

Chen, J.H. 2011. Petascale direct numerical simulation of turbulent combustion fundamental insights towards predictive models. Proc. Comb. Inst. 33, 99-123.

Chen, J.H., Echekki, T. \& Kollman, W. 1998 The mechanism of two-dimensional pocket formation in lean premixed methane air flames with implications for turbulent combustion. Combust. Flame 116, 15-48.

Chen, J.H. and Im, H.G., 1998, "Correlation of Flame Speed with Stretch in Turbulent Premixed Methane/Air Flames," 27th International Symposium on Combustion, The Combustion Institute, vol. 27, pp. 819-826.

Chen, J.H. and Im, H.G., 2000, "Stretch Effects on the Burning Velocity of Turbulent Premixed Hydrogen-Air Flames," Proceedings of the Combustion Institute, 28, pp. 211218.

Coppola, G., Coriton, B., Gomez, A. 2009. Highly turbulent counterflow flames: a laboratory scale benchmark for practical systems. Comb. Flame, 156, 1834-1843.

Cuenot, B, Poinsot, T. 1994. Effects of curvature and unsteadiness in diffusion flames. Implications for turbulent diffusion combustion. Proc. Symp. Int. Combust., 25th, Irvine, pp. 1383-90.

Dabireau, F., Cuenot, B., Vermorel, O., Poinsot, T. 2003. Interaction of flames of $\mathrm{H} 2+\mathrm{O} 2$ with inert walls, Comb. Flame, 135, 123-133.

Desjardins, O, Moureau, V., Pitsch, H. 2008. An accurate conservative level set/ghost fluid method for simulating turbulent atomization. J. Comp. Phys. 18, 8395-8416.

Domingo, P., Vervisch, L. 1996 Triple flames and partially premixed combustion in autoignition of non-premixed mixtures. 26th Symp. (Int.) on Combustion, The Combustion Institute, Pittsburgh, 233-240.

Driscoll, J.F. 2008. Turbulent premixed combustion: flamelet structure and its effect on turbulent burning velocities. Prog. Energy Combust. Sci., 34, 91-134.

Echekki, T., Chen, J.H. 1996. Unsteady strain rate and curvature effects in turbulent premixed methane-air flames. Combust. Flame 106, 184-202.

Echekki, T., Chen, J.H. 1998. Structure and propagation of methanol-air triple flames. Comb. Flame. 114, 231-245.

Egolfopoulos, F.N., Campbell, C.S. 1996. Unsteady counterflowing strained diffusion flames: diffusion-limited frequency response. J. Fluid Mech., 318, 1-29.

Eswaran, V., Pope, S. 1988. An examination of forcing in direct numerical simulations of turbulence. Computational Fluids , 16 (3), 257-278.

Favier, V., Vervisch, L. 2001. Edge flames and partially premixed combustion in diffusion flame quenching. Comb. Flame, 125, 788-803.

Grout R.W., Gruber A., Kolla H., Bremer P.-T., Bennett J.C., Gyulassy A., Chen J.H. 2012. A direct numerical simulation study of turbulence and flame structure in transverse jets analysed in jet-trajectory based coordinates, J. Fluid Mech. 706(10), pp. 351-383.

Gruber, A., Sankaran, R., Hawkes, E.R., Chen, J.H. 2010. Turbulent flame-wall interaction: a direct numerical simulation study. J. Fluid Mech. 658, 5-32.

Gruber, A., Chen, J.H., Valiev, D., Law, C.K. 2012. Direct numerical simulation of premixed flame boundary layer flashback in turbulent channel flow. J. Fluid Mech., 709, 516-542.

Hamlington, P.E., Poludnenko, A.Y., and Oran, E.S. 2011. Interactions between turbulence and flames in premixed reacting flows. Phys. Fluids, 23, 125111. 
Hamlington, P.E., Poludnenko, A.Y., and Oran, E.S. 2012. Intermittency in premixed turbulent reacting flows. Phys. Fluids, 24, 075111.

Hawkes, E.R., Chatakonda, O., Kolla, H., Kerstein, A.R., and Chen, J.H. 2012. A petascale direct numerical simulation study of the modelling of flame wrinkling for large-eddy simulations in intense turbulence. Combust. Flame, 159, 2690-2703.

Hawkes, E.R., Sankaran, R., Chen, J.H., Kaiser, S.A., Frank, J.H. 2009. An analysis of lowerdimensional approximations to the scalar dissipation rate using direct numerical simulations of plane jet flames. Proc. Comb. Inst., 32, 1455-1463.

Haworth, D., Cuenot, B., Poinsot, T. \& Blint, R. 2000 Numerical Simulation of turbulent propane-air combustion with non homogeneous reactants. Combust. Flame 121, 395-417.

Hilbert, R., Thevenin, D. 2002. Autoignition of turbulent non-premixed flames investigated using direct numerical simulations. Comb. Flame. 128, 22-37.

Hilbert, R., Tap, F., El-Rabii, H., and Thévenin, D. 2004. Impact of detailed chemistry and transport models on turbulent combustion simulations. Prog. Energy Combust. Sci., 30, 61-117.

Huan, X., Marzouk, Y. M. 2013. Simulation-based optimal Bayesian experimental design for nonlinear systems. J. Comp. Phys. 232 (1), 288-317.

Im, H. G., Arias, P. G., Chaudhuri, S., Uranakara, H., 2016, Direct Numerical Simulations of Statistically Stationary Turbulent Premixed Flames, Combustion Science and Technology, 188 (8), 1182-1198.

Im, H. G., Bechtold, J. K., Law, C. K., 1995, Counterflow diffusion flames with unsteady strain rates," Combustion Science and Technology, 106, pp. 345-361.

Im, H. G., Chen, J. H., and Law, C. K., 1998, Ignition of Hydrogen/Air Mixing Layer in Turbulent Flows. 27th International Symposium on Combustion, The Combustion Institute, vol. 27, pp. 1047-1056.

Im, H. G., and Chen, J. H., 1999, Structure and Propagation of Triple Flames in Partially Premixed Hydrogen/Air Mixtures, Combustion and Flame, 119, pp. 436-454.

Im, H. G. and Chen, J. H., 2001, Effects of Flow Strain on Triple Flame Propagation, Combustion and Flame, 126, pp. 1384-1392.

Jenkins, K. W., and Cant, R. S. 2002. Curvature effects on flame kernels in a turbulent environment. Proc. Combust. Inst., 29, 2023-2029.

Jenkins, K. W., Klein, M., Chakraborty, N., and Cant, R. S. 2006. Effects of strain rate and curvature on the propagation of a spherical flame kernel in the thin-reactionzones regime. Combust. Flame, 145, 415-434.

Jimenez, C., Cuenot, B., Poinsot, T. \& Haworth, D. 2002 Numerical simulation and modeling for lean stratified propane-air flames. Combust. Flame 128, 1-21.

Kee, R.J. Rupley, F.M., Miller, J.A. 1989. Chemkin-II: A Fortran chemical kinetics package for the analysis of gas-phase chemical kinetics, Sandia Report SAND-89-8009.

Kim J, Moin P, Moser RD. 1987. Turbulence statistics in fully-developed channel flow at low Reynolds number. J. Fluid Mech. 177:133-66.

Kim, Y.J., Lee, B.J., Im, H.G. 2017. Scale effect on dynamics of meso-scale bluff-bodystabilized flames in lean premixed hydrogen-air and syngas-air mixtures. Fourteenth International Conference on Flow Dynamics, Sendai, Japan, Nov. 1-3, 2017.

Lapointe, S., Savard, B., \& Blanquart, G. 2015. Differential diffusion effects, distributed burning, and local extinctions in high Karlovitz premixed flames. Combustion and Flame , 162 (9), 3341-3355.

Le Maître, O.P.; Knio, O.M. 2010. Spectral Methods for Uncertainty Quantification: with Applications to Computational Fluid Dynamics, Springer. 
Lebas, R., Menard, T., Beau, P.A., Berlemont, A., Demoulin, F.X. 2009. Numerical simulation of primary break-up and atomization: DNS and modelling study. Int. J. Multiphase Flow, 35, 247-260.

Lee, E.D., Yoo, C.S., Chen, J.H., Frank, J.H. 2010. Effect of NO on extinction and re-ignition of vortex-perturbed hydrogen flames. Comb. Flame, 157, 217-229.

Lee, S., Lele, S.K., Moin, P. 1991. Simulations of spatially decaying compresible turbulence. Center for Turbulence Research, NASA Ames/Stanford University, Manuscript 126.

Lignell, D.O., Chen, J.H., Smith, P.J., Lu, T., Law, C.K. 2007. The effect of flame structure on soot formation and transport in turbulent nonpremixed flames using direct numerical simulation. Comb. Flame, 151, 2-28.

Lignell, D.O., Chen, J.H., Smith, P.J. 2008. Three-dimensional direct numerical simulation of soot formation and transport in a temporally evolving nonpremixed ethylene jet flame. Comb. Flame, 155, 316-333.

Lipatnikov, A.N., and Chomiak, J. 2002. Turbulent flame speed and thickness: phenomenology, evaluation, and application in multi-dimensional simulations. Prog. Energy Combust. Sci., 28, 1-74.

Lipatnikov, A.N., and Chomiak, J. 2005. Molecular transport effects on turbulent flame propagation and structure. Prog. Energy Combust. Sci., 31, 1-73.

Lipatnikov, A.N., and Chomiak, J. 2010. Effects of premixed flames on turbulence and turbulent scalar transport. Prog. Energy Combust. Sci., 36, 1-102.

Liu, C.C., Shy, S.S., Peng, M.W., Chiu, C.W., and Dong, Y.-C. 2012. High-pressure burning velocities measurements for centrally-ignited premixed methane/air flames interacting with intense near-isotropic turbulence at constant Reynolds numbers. Combust. Flame, 159, 2608-2619.

Lu, T.F., Yoo, C.S., Chen, J.H., Law, C.K. 2010. Three-dimensional direct numerical simulation of a turbulent lifted hydrogen jet flame in a heated coflow: a chemical explosive mode analysis. J. Fluid Mech., 652, 45-64.

Mahalingam, S., Chen, J.H. and Vervisch, L. 1995. Finite-Rate Chemistry and Transient Effects in Simulations of Turbulent Non-premixed Flames, Combustion and Flame, 102: 285.

Mastorakos, E., Baritaud, T.A., Poinsot, T.J. 1997. Numerical simulations of autoignition in turbulent mixing flows. Comb. Flame, 109, 198-223.

Matalon, M. 1983. On flame stretch. Comb. Sci. Tech. 31, 169-181.

Mashayek, F. 1998. Droplet-turbulence interactions in low Mach number homogeneous shear two-phase flows. J. Fluid Mech. 367:163-203.

Minamoto, Y., Fukushima, N., Tanahashi, M., Miyauchi, T., Dunstan, T.D., and Swaminathan, N. 2011. Effect of flow-geometry on turbulence-scalar interaction in premixed flames. Phys. Fluids, 23, 125107.

Minamoto, Y, Swaminathan, N., Cant, R.S., and Leung, T. 2014. Reaction zones and their structure in MILD combustion. Combust. Sci. Tech., 186(8), 1075-1096.

Mizobuchi, Y., Tachibana, S., Shinjo, J., Ogawa, S., Takeno, T. 2002. A numerical analysis of the structure of a turbulent hydrogen jet lifted flame. Proc. Comb. Inst., 29, 2009-2015.

Mizobuchi, Y., Shinjo, J., Ogawa, S., Takeno, T. 2005. A numerical study on the formation of diffusion flame islands in a turbulent hydrogen jet lifted flame. Proc. Comb. Inst., 30, 611-619.

Modest, M.F. 2013. Radiative Heat Transfer, 3rd ed., Academic Press.

Moin, P., Mahesh, K. 1998. Direct numerical simulation: a tool in turbulence research. Ann. Rev. Fluid Mech. 40, 539-578.

Mueller, M.E., Blanquart, G., Pitsch, H. 2009. Hybrid method of moments for modeling soot formation and growth. Comb. Flame, 156, 1143-1155. 
Najm, H.N., Knio, O.M., Paul, P.H., Wyckoff, P.S. 1998. A study of flame observables in premixed methane-air flames. Comb. Sci. Tech. 140, 369-403.

Nikolaou, Z.M., Swaminathan, N. 2015. Direct numerical simulation of complex fuel combustion with detailed chemistry: physical insight and mean reaction rate modeling. Comb. Sci. Tech. 187, 1759-1789.

O'Brien, J.Towery, C.A.Z., Hamlington, P.E., Ihme, M., Poludnenko, A.Y., Urzay, J. 2017. The cross-scale physical-space transfer of kinetic energy in turbulent premixed flames. Proc. Comb. Inst. 36, 1967-1975.

Pascucci, V.; Scorzelli, G.; Summa, B.; Bremer, P. T.; Gyulassy, A.; Christensen, C.; Philip, S.; Kumar, S. The ViSUS Visualization Framework, Chapter 19, pp. 401-414. Chapman \& Hall/CRC Computational Science, 2012.

Peters, N. Turbulent Combustion, Cambridge University Press, 2000.

Poinsot, T. 1996. Using direct numerical simulations to understand premixed turbulent combustion. Proc. Combust. Inst., 26, 219-232.

Poinsot, T., Candel, S., and Trouvé, A. 1995. Applications of direct numerical simulation to premixed turbulent combustion. Prog. Energy Combust. Sci, 21, 531-576.

Poinsot, T., Veynante, D. 2005. Theoretical and numerical combustion. 2nd ed. RT Edwards, Inc.

Poinsot, T., Veynante, D., Candel, S. 1991. Quenching processes and premixed turbulent combustion diagrams. J. Fluid Mech., 228, 561-606.

Poinsot, T., Haworth, D.C., Bruneaux, G., 1993. Direct simulation and modeling of flamewall interaction for premixed turbulent combustion, Comb. Flame, 95, 118-132.

Pope, S.B. 1987. Turbulent premixed flames. Ann. Rev. Fluid Mech., 19, 237-270.

Rogallo RS. 1981. Numerical experiments in homogeneous turbulence. NASA TM-81315

Rogallo, R.S, Moin, P. 1984. Numerical simulation of turbulent flows. Annu. Rev. Fluid Mech. 16:99-137

Ronney, P.D. 1995. Modeling in Combustion Science. Lecture Notes in Physics, Volume 449, 1-22.

Rutland, C.J., Ferziger, J.H. 1991. Simulations of flame-vortex interactions. Comb. Flame. 84, 343-360.

Rutland, C.J., Cant, R.S. 1994. Turbulent transport in premixed flames. In Proc. Summer Program Center for Turbulence Research, NASA Ames/Stanford University.

Salenbauch, S., Sirignano, M., Marchisio, D., Pollack, M., D’Anna, A., Hasse, C. 2017. Detailed particle nucleation modeling in a sooting ethylene flame using a Conditional Quadrature Method of Moments (CQMOM), Proc. Comb. Inst., 36:771-779.

Sankaran, R., Hawkes, E.R., Chen, J.H., Lu, T.F., Law, C.K. 2007. Structure of a spatially developing turbulent lean methane-air Bunsen flame. Proc. Comb. Inst. 27, 1291-1298.

Sankaran, R., Hawkes, E.R., Yoo, C.S., Chen, J.H. 2015. Response of flame thickness and propagation speed under intense turbulence in spatially developing lean premixed methane-air jet flames. Comb. Flame, 162, 3294-3306.

Sarkar S, Erlebacher G, Hussaini M.Y. 1991. Direct simulation of compressible turbulence in a shear flow. Theor. Comput. Fluid Dyn. 2:291-305.

Savard, B., and Blanquart, G. 2015. Broken reaction zone and differential diffusion effects in high Karlovitz n-C7H16 premixed turbulent flames. Combust. Flame, 162, 2020-2033.

Savard, B., Bobbitt, B., Blanquart, G. 2015. Structure of a high Karlovitz n-C7H16 premixed turbulent flame. Proc. Combust. Inst., 35, 1377-1384.

Shim, Y.S., Fukushima, N., Shimura, M., Nada, Y., Tanahashi, M., Miyauchi, T. 2013. Radical fingering in turbulent premixed flame classified into thin reaction zones. Proc. Combust. Inst., 34, 1383-1391. 
Tanahashi, M., Nada, Y., Ito, Y., Miyauchi, T. 2002. Local flame structure in the well-stirred reactor regime. Proc. Combust. Inst., 29, 2041-2049.

Taylor, G.I. 1938. The spectrum of turbulence. Proc. R. Soc. Lond. 164 (919), 476-490.

Tomboulides, A. 2013. DNS of Flame Propagation Phenomena, ERCOFTAC Spring Festivl, Toulon.

Trouve, A., Poinsot, T. 1994. The evolution equation for the flame surface density in turbulent premixed combustion. J. Fluid Mech. 278, 1-31.

Vervisch, L., Hauguel, R., Domingo, P, Rullaud, M. 2004. Three facets of turbulent combustion modelling: DNS of premixed V-flame, LES of lifted nonpremixed flame and RANS of jet-flame. J. Turbulence, 5, 004.

Wabel, T.M. Skiba, A.W., Temme, J.E., Driscoll, J.F. 2017. Measurements to determine the regimes of premixed flames in extreme turbulence. Proc. Comb. Inst. 36, 1809-1816.

Wacks, D. H., Chakraborty, N., Klein, M., Arias, P. G., Im, H. G., 2016, "Flow Topologies in Different Regimes of Premixed Turbulent Combustion: A Direct Numerical Simulation Analysis," Physical Review Fluids, 1, 083401.

Wang, H., Hawkes, E.R., Chen, J.H. 2017. A direct numerical simulaiton study of flame structure and stabilization of an experimental high $\mathrm{Ka}$ CH4/air premixed jet flame. Comb. Flame, 180, 110-123.

Williams, F.A. 1985. Combustion Theory, 2nd ed., Westview Press.

Yoo, C. S. and Im, H. G., 2005, Transient Dynamics of Edge Flames in a Laminar Nonpremixed Hydrogen-Air Counterflow, Proceedings of the Combustion Institute, 30, 349-356.

Yoo, C. S. and Im, H. G., 2007. Transient Soot Dynamics in Turbulent Nonpremixed Ethylene-Air Counterflow Flames. Proceedings of the Combustion Institute, 31, 701-708.

Yoo, C.S., Richardson, E.S., Sankaran, R., Chen, J.H. 2011. A DNS study on the stabilization mechanism of a turbulent lifted ethylene jet flame in highly heated coflow. Proc. Comb. Inst., 33, 1619-1627. 